\title{
SERPÚLIDOS (ANNELIDA, SERPULIDAE) COLECTADOS EN LA CAMPAÑA OCEANOGRÁFICA "FAUNA II" Y CATÁLOGO ACTUALIZADO DE ESPECIES ÍBERO-BALEARES DE LA FAMILIA SERPULIDAE
}

\author{
Jesús Alcázar* \& Guillermo San Martín \\ Departamento de Biología (Zoología), Facultad de Ciencias, Universidad Autónoma de \\ Madrid, calle Darwin, 2, Canto Blanco, 28049 Madrid, España. \\ *Dirección para la correspondencia: jalcazar.1bach@gmail.com
}

\section{RESUMEN}

Se presentan los resultados de la identificación del material de la familia Serpulidae (Polychaeta) recolectado en la campaña oceanográfica Fauna II, así como la revisión de citas de presencia íbero-balear desde el catálogo de poliquetos más reciente (Ariño, 1987). Se identificaron 16 especies pertenecientes a 10 géneros, además de la primera cita íbero-balear de una quimera bioperculada (Ten Hove \& Ben-Eliahu, 2005) de la especie Hydroides norvegicus Gunnerus, 1768. En cuanto a la revisión del catálogo se mencionan 65 especies, actualizando el nombre de 20 de ellas y añadiendo cinco especies ausentes en el catálogo de Ariño (1987): Hydroides stoichadon Zibrowius, 1971, Laeospira corallinae (de Silva \& Knight-Jones, 1962), Serpula cavernicola Fassari \& Mòllica, 1991, Spirobranchus lima (Grube, 1862) y Spirorbis inornatus L'Hardy \& Quièvreux, 1962. Se cita por primera vez Vermiliopsis monodiscus Zibrowius, 1968 en el Atlántico ibérico y a partir de la bibliografia consultada, se muestra la expansión en la distribución íbero-balear de Hydroides dianthus (Verrill, 1873) y de H. stoichadon.

Palabras clave: Serpulidae; Polichaeta; Península Ibérica; catálogo de especies; quimera bioperculada; íbero-balear.

\section{ABSTRACT}

Serpulids (Annelida, Serpulidae) collected in the oceanographic cruise "Fauna II" and the updated species catalogue for the family Serpulidae in the iberian-balearic coasts

In this paper, the results of the identification of the specimens of the Family Serpulidae (Polychaeta) collected during the Cruise Fauna // and a bibliographic review of the presence of Serpulidae along the IberianBalearic coasts since the last complete catalogue of Iberian polychaetes (Ariño, 1987) are presented. A total of 16 species belonging to 10 genera were identified, in addition to the first bioperculate chimaera (Ten Hove \& Ben-Eliahu, 2005) of the species Hydroides norvegicus Gunnerus, 1768, reported for the Iberian-Balearic coasts. As for the catalogue revision a total of 65 species are reported, 20 of which have their real name emended. Five of the species found in the updated bibliography are new for the lberian-Balearic coasts: Hydroides stoichadon Zibrowius, 1971, Laeospira corallinae (de Silva \& Knight-Jones, 1962), Serpula cavernicola Fassari \& Mòllica, 1991, Spirobranchus lima (Grube, 1862) and Spirorbis inornatus L'Hardy \& Quièvreux, 1962. Vermiliopsis monodiscus Zibrowius, 1968 is reported for the lberian Atlantic coasts for the first time, and from the literature the expansion of the Iberian-Balearic distribution of Hydroides dianthus (Verrill, 1873) and $H$. stoichadon is shown.

Key words: Serpulidae; Polichaeta; Iberian Peninsula; species catalogue; bioperculate chimaera; Iberian-Balearic.

Recibido/Received: 19/04/2014; Aceptado/Accepted: 16/09/2016; Publicado en línea/Published online: 11/11/2016

Cómo citar este artículo/Citation: Alcázar, J. \& San Martín, G., 2016. Serpúlidos (Annelida, Serpulidae) colectados en la Campaña Oceanográfica "Fauna II" y catálogo actualizado de especies íbero-baleares de la familia Serpulidae. Graellsia, 72(2): e053. http://dx.doi.org/10.3989/graellsia.2016.v72.120 


\section{Introducción}

La familia Serpulidae Rafinesque, 1815, está constituida por anélidos poliquetos que viven alojados en tubos de carbonato cálcico que ellos mismos producen gracias a las glándulas tubulares del collar (Zibrowius, 1968). Las especies que conforman esta familia se caracterizan por presentar una corona branquial y porque, en la mayoría de los taxones, uno de los filamentos branquiales que la forman se diferencia en un opérculo que sella la entrada del tubo cuando el animal se retrae en el mismo y cuya forma es utilizada como un carácter taxonómicamente identificativo, junto con las sedas del primer segmento torácico o sedas del collar (Bianchi, 1981; Bastida-Zavala, 2009). En algunas especies se han descrito ejemplares que poseían dos opérculos, denominados bioperculados (Okada, 1933). También se ha registrado un fenómeno conocido como quimera bioperculada, cuando los serpúlidos presentan dos opérculos de distinta morfología, siendo uno el típico de la especie y el otro posiblemente un estado ontogenético transitorio (Ten Hove \& Ben-Eliahu, 2005).

Esta familia de anélidos poliquetos queda actualmente comprendida en el clado Palpata-CanalipalpataSabellida (Rouse, 2001). La sistemática de la familia Serpulidae ha sufrido distintas modificaciones a lo largo de los años a medida que se realizaban estudios basados en caracteres moleculares frente a la sistemática más tradicional, basada únicamente en caracteres morfológicos. Estos trabajos corroboraron la monofilia de Serpulidae si bien se modificó la clasificación de la subfamilia Fabriciinae Rioja, 1923, dentro de Sabellidae Latreille, 1825, para mantener los tres clados monofiléticos, quedando Serpulidae como grupo hermano de Fabriciidae, y ambos hermanos de Sabellidae (Kupriyanova y Rouse, 2008).

Tradicionalmente Serpulidae incluía a las subfamilias Serpulinae Rafinesque, 1815, Filograninae Rioja, 1923 y Spirorbinae Chamberlin, 1919, si bien esta última pasó a ser considerada como Familia Spirorbidae, grupo hermano de Serpulidae (Pillai, 1970). Sin embargo, Fitzhuhg (1989) demostró que separar Spirorbidae de la familia Serpulidae hacía a ésta parafilética mientras que la inclusión de Spirorbinae de nuevo como subfamilia dentro de Serpulidae mantenía a estos últimos como grupo monofilético. Posteriores estudios realizados por Lehrke et al. (2007) corroboraron la posición de Spirorbinae dentro de Serpulidae aunque no avalaban las otras dos subfamilias tradicionales, sino que redistribuían los serpúlidos en cuatro grupos monfiléticos: Spirorbinae, el grupo Serpula, el grupo Protula y el grupo Pomatoceros. Es por ello que podemos afirmar que esta familia se encuentra pendiente de una importante revisión a nivel de subfamilias y complejos de géneros (Ten Hove y Kupriyanova, 2009).

El catálogo de poliquetos ibéricos sedentarios realizado por Ariño (1987) incluía a esta familia, en aquel entonces considerada como dividida en Serpulidae y Spirorbidae. Sin embargo, planteamos como idea de partida para este estudio que desde entonces la diversidad específica en nuestras costas puede haber aumentado. Posibles explicaciones a este aumento serían un mayor estudio de esta familia en las costas ibéricas, así como el fenómeno del fouling u otros que permitan la dispersión de especies por actividades humanas. El fouling se trata de un mecanismo de dispersión artificial por el cual especies acuáticas sésiles como los serpúlidos pueden establecerse sobre superficies artificiales sumergidas, como cascos de embarcaciones, y reproducirse de forma exitosa, de modo que serían capaces de establecer colonias a lo largo del recorrido de estos buques (Emara \& Belal, 2004). Por tanto cabe esperar que la distribución de especies de serpúlidos por el ámbito íbero-balear haya aumentado, además de la posibilidad de encontrar especies alóctonas o desconocidas hasta la fecha en nuestras costas.

Con el fin de presentar un catálogo de serpúlidos íbero-baleares actualizado respecto a los listados de poliquetos ibéricos ya publicados (Campoy, 1982; Ariño, 1987) y poder así hacer en el futuro un seguimiento de la posible introducción de especies alóctonas de esta familia en nuestras costas hemos llevado a cabo una revisión bibliográfica exhaustiva sobre la misma en dicha área. Además, complementariamente se identificaron muestras de unos 50 lotes pertenecientes a la Campaña Fauna II (año 1991) del Proyecto Fauna Ibérica, conservadas por el Museo Nacional de Ciencias Naturales de Madrid (MNCN). Este estudio pretende servir así también como base para la realización de un capítulo nuevo, en este caso de serpúlidos de un futuro nuevo volumen de Poliquetos del proyecto "Fauna Ibérica".

Se presentan por tanto dos conjuntos de resultados bien diferenciados: las especies identificadas a partir del estudio de las muestras de la campaña Fauna II y por otro lado la actualización del catálogo de serpúlidos íbero-baleares.

\section{Material y métodos}

La campaña Fauna II se desarrolló en julio de 1991 frente a las costas de Galicia y de la cornisa cantábrica. Las muestras estudiadas se obtuvieron mayoritariamente mediante arrastres bentónicos con Bou de Varas entre 25 y $770 \mathrm{~m}$ de profundidad. Los datos de dichas muestras se presentan en el Apéndice 1. Los ejemplares analizados se encontraban conservados en etanol al 70\%. Fueron identificados en el laboratorio de Biología Marina e Invertebrados de la Universidad Autónoma de Madrid, y posteriormente depositados de nuevo en el Museo Nacional de Ciencias Naturales de Madrid. La imagen presentada se realizó con una cámara Nikon D5100 acoplada a una lupa Nikon SMZ-1500.

La actualización del catálogo de Ariño (1987) se llevó a cabo mediante revisión bibliográfica, 
anotando todas las citas encontradas que demuestren la presencia de Serpulidae en el ámbito íbero-balear, considerando las costas de la península Ibérica, el archipiélago Balear, las islas atlánticas de las costas de Galicia, la isla de Alborán, las islas Chafarinas y las islas Columbretes, así como el citado estudio de los ejemplares de Fauna II.

Debido a las razones ya esgrimidas anteriormente, hemos considerados sólo dos subfamilias dentro de los serpúlidos: Serpulinae y Spirorbinae. Los géneros y especies se han ordenado alfabéticamente y cada especie aparece acompañada de citas bibliográficas en las cuales se ha referido su presencia en el ámbito íbero-balear, y un asterisco $(*)$ si la especie ha sido identificada durante este estudio. Las citas han sido referenciadas en formato numérico y se pueden consultar en el Apéndice 2.

A continuación se enumeran todas las localidades íbero-baleares recogidas, agrupadas en sus respectivos mares y ordenadas siguiendo la línea de costa desde el País Vasco hacia el oeste, bordeando todo el ámbito íbero-balear, y terminando en las costas de Cataluña. Aparecen subrayadas las localidades correspondientes a los lotes de la campaña Fauna II (Apéndice 1), como localidades que se adicionan al catálogo.

Cabe destacar que según los trabajos consultados algunas localidades son muy generales (ej.: "Galicia") mientras que en otras la información es mucho más específica (ej.: "Ensenada de Baiona, Ría de Vigo").

Por último, se especifica qué especies se adicionan al listado de poliquetos ibéricos de (Ariño, 1987).

La nomenclatura empleada para este trabajo se basa principalmente en Ten Hove \& Kupriyanova (2009) si bien ha sido actualizada según la información presente en la base WoRMS (World Register of Marine Species) y la Tesis Doctoral de Gil (2011).

\section{Resultados}

\section{ESPECIES IDENTIFICADAS DE LA CAMPaña Fauna //}

A continuación se enumeran los géneros y especies identificados y ordenados alfabéticamente. Para cada especie se presenta el código de la estación donde se tomó la muestra y entre paréntesis el número de ejemplares. Con una almohadilla (\#) se indican los ejemplares de Hydroides norvegicus Gunnerus, 1768, identificados como quimeras bioperculadas. En el Apéndice 1 pueden consultarse otros datos de las estaciones, como nombre de la localidad, coordenadas, profundidad y método de muestreo.

Para identificar la especie a la que pertenece cada ejemplar se utilizaron distintas claves y descripciones, tanto claves de géneros (Bianchi, 1981; Ten Hove \& Kupriyanova, 2009; Fauchald, 1977; Bastida-Zavala, 2009) como de especies, en este caso principalmente la propuesta por Bianchi (1981), y en menor medida la de Bastida-Zavala (2009).
Para llevar a cabo estas identificaciones se tuvieron en cuenta los caracteres taxonómicamente más relevantes para esta familia siguiendo las claves mencionadas, como el tamaño del animal, la presencia o ausencia de opérculo, así como su morfología y la del pedúnculo opercular, la morfología de las sedas del collar, y la morfología del tubo.

A continuación se recoge un total de 16 especies agrupadas en 10 géneros.

Familia Serpulidae Rafinesque, 1815

Subfamilia Serpulinae Rafinesque, 1815

Género Apomatus Philippi, 1844

Apomatus similis Marion \& Bobretzky, 1875

145A (2)

Género Ditrupa Berkeley, 1835

Ditrupa arietina (O. F. Müller, 1776)

111A (8), 124A (1), 153A (4)

Género Hyalopomatus Marenzeller, 1878

Hyalopomatus marenzelleri Langerhans, 1884

173A (1)

Género Hydroides Gunnerus, 1768

Hydroides dianthus (Verrill, 1873)

158A (1)

Hydroides elegans (Haswell, 1883) 104P (1)

Hydroides norvegicus Gunnerus, 1768

85DH (1), 91A (7), \#98A (11), 105A (1), 106A (1), 108A (7), 109A (1), 113A (3), 114A (6), 123A (1), 128A (1), 141A (1), \#142A (3), 143A (11), 145A (1), 148A (4), 150A (1), 151A (5), 152A (1), 153A (1), 156A (3), 164A (1), 168A (1), 170A (9)

Algunos individuos de los lotes 98A (Fig. 1) y 142A, constituyen la primera cita de quimeras bioperculadas de esta especie en nuestra región, y probablemente en todo el mundo, ya que Ten Hove \& Ben-Eliahu (2005) consideran que los individuos citados anteriormente como quimeras bioperculadas, pertenecen realmente a la especie Hydroides elegans. Se han examinado las sedas del collar y se ha comprobado que corresponden a la morfología típica de $H$. norvegicus: un par de gruesos dientes en la base de la prolongación más distal frente a la presencia de varios dientes de pequeño tamaño en la especie próxima H. elegans (Bianchi, 1981).

\section{Hydroides stoichadon Zibrowius, 1971 128A (1)}

Citada únicamente en el ámbito mediterráneo, en las costas italianas y del sureste francés (Ten Hove y Kupriyanova, 2009) y de Almería (San Martín et al., 1990), por lo tanto el ejemplar identificado se trata de la segunda cita de presencia en el ámbito íbero-balear y primera cita para la costa cantábrica (Cabo Mayor). 


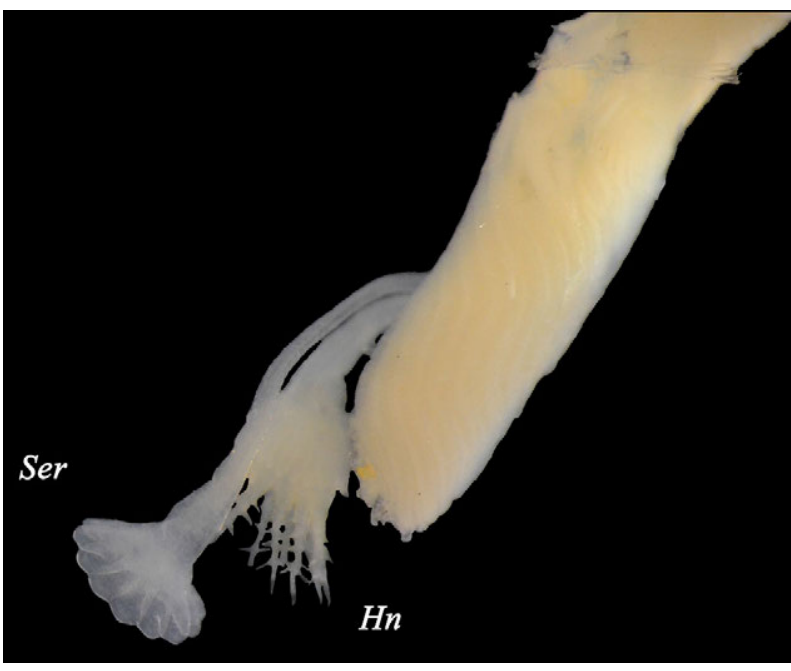

Fig. 1.- Quimera bioperculada de Hydroides norvegicus (lote 98A Proyecto Fauna Ibérica II). Detalle de los dos opérculos sobre la corona branquial. Se pueden diferenciar el opérculo típico de H. norvegicus (indicado como " $\mathrm{Hn}$ ") del opérculo tipo Serpula (indicado como "Ser"). Escala: $1 \mathrm{~mm}$.

Fig. 1.- Bioperculate chimaera of Hydroides norvegicus (lot 98A of the "Proyecto Fauna Ibérica II"). Detail of two opercula on the gill crown. The operculum of $H$. norvegicus (indicated as "Hn") can be easily differenciated from the Serpula operculum type (indicated as "Ser"). Scale: $1 \mathrm{~mm}$.

Género Janita Saint Joseph, 1894

Janita fimbriata (Delle Chiaje, 1822) 158A (1)

Género Placostegus Philippi, 1844

Placostegus tridentatus (Fabricius, 1779) 113A (1), 128A (1)

Género Pomatoceros Philippi, 1844

Pomatoceros lamarcki (Quatrefages, 1866) 131A (2)

Pomatoceros triqueter (Linnaeus, 1758) 141A (1)

Género Serpula Linnaeus, 1758

Serpula concharum Langerhans, 1880 98A (5), 101A (1), 105A (1), 108A (29), 112DH (1), $113 \mathrm{~A}(5), 114 \mathrm{~A}(2), 134 \mathrm{~A}(2), 148 \mathrm{~A}(21), 150 \mathrm{~A}(2), 151 \mathrm{~A}$ (1), 156A (9), 162A (3), 163A(1), 170A(1), 171A(3)

Serpula lobiancoi Rioja, 1917 158A (2)

Serpula vermicularis Linnaeus, 1767 91A (1), 112DH (1), 113A (4), 123A (1), 128A (3), 131A (1), 145A (1), 152A (2), 162A (1), 168A (1), 173A (1)

Género Vermiliopsis Saint-Joseph, 1894

Vermiliopsis infundibulum (Philippi, 1844) 128A (1), 158A (1), 171A (1)

Vermiliopsis monodiscus Zibrowius, 1968 173A (5)

\section{Catálogo de los Serpúlidos íbero-baleares}

A continuación se presenta el catálogo completo de citas de serpúlidos íbero-baleares. Para conformar dicho catálogo se tomaron como base los listados de poliquetos ibéricos de Campoy (1982) y Ariño (1987), a los cuales se añadieron las citas de presencia de serpúlidos pertenecientes a trabajos posteriores a dichos catálogos en el ámbito íbero-balear, como se ha comentado, así como las especies identificadas en este estudio a partir del material cedido por el Museo.

En el catálogo que se presenta a continuación se reúne un total de 32 géneros y 64 especies de distribución íbero-balear. De éstas, 20 especies nunca habían sido citadas para las costas íbero-baleares con su nombre actualmente válido. Además, como resultado de la revisión bibliográfica, se incluyen citas de cuatro especies como adiciones al catálogo de poliquetos ibéricos de Ariño (1987).

Familia Serpulidae Rafinesque, 1815

Subfamilia Serpulinae Rafinesque, 1815

Género Apomatus Philippi, 1844

Apomatus ampulliferus Philippi, 1844

Referencias: 36, 39, 69, 118, 126, 130, 131, 169.

Citado en algunos casos como Protula lusitanica McIntosh, 1885.

Localidades citadas: Cantábrico; Mediterráneo (Bahía de Palma de Mallorca).

Apomatus similis Marion \& Bobretzky, 1875 (*)

Referencias: 15, 29, 30, 37, 69, 118, 121, 125, 126, 129, 130, 135, 147, 166.

Localidades citadas: Cantábrico [Cierzo, Santander, San Vicente de la Barquera (Cantabria), Gijón]; Atlántico (Cabo Vilán, A Coruña, Portugal]; Mediterráneo [Valencia, Mahón (Menorca)].

Género Bathyvermilia Zibrowius, 1973

Bathyvermilia eliasoni (Zibrowius, 1970)

Referencias: 37, 39, 167.

Citado siempre como Vermiliopsis eliasoni Zibrowius, 1967.

Localidades citadas: Atlántico (costa ibérica de Portugal).

Género Ditrupa Berkeley, 1835

Ditrupa arietina (O. F. Müller, 1776) (*)

Referencias: 2, 3, 5, 14, 15, 29, 30, 36, 37, 38, 39, 47, 48, 62, 69, 116, 125, 126, 130, 134, 138, 147, 152, 159.

Localidades citadas: Cantábrico (Ondarroa, País Vasco, Ribadesella, Cabo de Peñas); Atlántico (Galicia, costa ibérica de Portugal); Mediterráneo [Isla de Alborán (Almería), Valencia, Mallorca, Islas Columbretes (Castellón), Cataluña].

Género Ficopomatus Southern, 1921

Ficopomatus enigmaticus (Fauvel, 1923)

Referencias: $2,3,36,37,38,39,42,44,49,50,52$, $53,68,69,70,83,128,130,133,155,164,165$. 
Citado en la mayoría de los casos como Mercierella enigmatica Fauvel, 1923.

Localidades citadas: Cantábrico [Guipúzcoa, Vizcaya (citada en ocasiones como País Vasco en general)]; Atlántico (Portugal); Mediterráneo [Mar Menor (Murcia), Gandía, Valencia, Albufera de Menorca, Barcelona].

Género Filograna Berkeley, 1835

Filograna implexa Berkeley, 1835

Referencias: 6, 9, 14, 34, 35, 36, 37, 38, 39, 48, 69, $85,86,88,98,99,101,111,116,118,119,123,125$, $126,129,130,134,137,138,144,147,150,151$, $159,166$.

Localidades citadas: Cantábrico (Santander, San Vicente de la Barquera); Atlántico [Fornelos, Batel y Segaño (estas tres últimas a veces citadas de forma genérica como Ría de Ferrol, o incluso incluyendo a toda la comunidad como Galicia), Arrábida (citado en ocasiones de forma genérica como costa ibérica de Portugal)]; Mediterráneo [Gibraltar, Mar de Alborán, Islas Chafarinas (Melilla), Valencia, Baleares, Islas Columbretes, Islas Medas (Gerona), Cabo de Creus (a veces citado de forma genérica como Gerona o incluso como Cataluña)].

Género Filogranula Langerhans, 1884

Filogranula annulata (O. G. Costa, 1861)

Referencias: 6, 9, 37, 39, 85, 86, 171.

Localidades: Mediterráneo [Gerona, Cabo de Creus (citado en ocasiones de forma genérica como Cataluña)].

Filogranula calyculata (O. G. Costa, 1861)

Referencias: 36, 37, 39, 167.

Citado como Omphalopoma cristata Langerhans, 1884.

Localidades citadas: Cantábrico [Cierzo (citado en ocasiones de forma genérica como Santander), Gijón]; Atlántico (costa ibérica de Portugal).

Filogranula gracilis Langerhans, 1884

Referencias: 36, 37, 39, 69, 167.

Citado como Omphalopoma gracilis (Langerhans, 1884).

Localidades citadas: Atlántico(costa ibérica dePortugal).

Género Hyalopomatus Marenzeller, 1878

Hyalopomatus marenzelleri Langerhans, 1884 (*)

Referencias: 37, 39, 167, 168.

Citado como Hyalopomatopsis marenzelleri (Langerhans, 1884).

Localidades citadas: Atlántico (Banco de Galicia, costa ibérica de Portugal).

Género Hydroides Gunnerus, 1768

Hydroides dianthus (Verrill, 1873) (*)

Referencias: 36, 37, 39, 41, 43, 77, 119, 126, 127,

$165,169,171$.

Localidades citadas: Cantábrico [Cabo de Oyambre (primera cita para las costas ibéricas cantábricas)]; Atlántico [Galicia, desembocadura del río Piedras
(Huelva)]; Mediterráneo [Mar de Alborán, Águilas (Murcia), Ensenada de los Alfaques (Tarragona), Blanes (Gerona)].

\section{Hydroides diramphus Mörch, 1863}

Referencias: 24, 36, 37, 39, 69, 125, 126, 130, 165 , 166, 169, 171.

Citado como Hydroides lunulifera o como Hydroides dirampha Mörch, 1863. Nunca ha sido citado para las costas íbero-baleares con el nombre actualmente válido.

Localidadescitadas:Mediterráneo(Valencia,Barcelona).

Hydroides elegans (Haswell, 1883) (*)

Referencias: 22, 23, 24, 25, 34, 36, 37, 38, 39, 41, 43, 77, 85, 86, 91, 92, 124, 165, 169, 171.

Citado en algunos casos como Serpula (Hydroides) elegans.

Localidades citadas: Atlántico [A Coruña, desembocadura del río Piedras (Huelva), Portugal]; Mediterráneo [Escombreras (Cartagena), Castellón, Ensenada de los Alfaques, Gerona, Blanes (en ocasiones citados de forma genérica como Cataluña)].

Hydroides helmatus (Iroso, 1921)

Referencias: 34, 37, 38, 39, 76, 85, 88, 142, 157.

Citado en la mayoría de referencias como Hydroides helmata (Iroso, 1921).

Localidades citadas: Mediterráneo [Mar de Alborán, Islas Chafarinas, Baleares, Cala Ganduf y L'Olla (estas últimas en ocasiones citadas de forma genérica como Isla de Cabrera), Gerona (en ocasiones citada de forma genérica como Cataluña)].

\section{Hydroides niger Zibrowius, 1971}

Referencias: 4, 71, 76, 88, 141, 146, 157.

Citado como Hydroides nigra Zibrowius, 1971.

Localidades citadas: Cantábrico (País Vasco); Atlántico (Portugal); Mediterráneo [Nerja (Málaga), Mar de Alborán, Almería, Islas Chafarinas, Murcia, Baleares].

\section{Hydroides norvegicus Gunnerus, 1768 (*)}

Referencias: 2, 3, 6, 9, 14, 15, 17, 20, 22, 24, 30, 31, $34,36,37,38,39,41,42,43,46,48,55,6,57,69,90$, $94,101,104,106,113,114,115,117,118,119,124$, 125, 126, 127, 129, 130, 131, 138, 147, 167, 169.

Citado en la mayoría de referencias como Hydroides norvegica Gunnerus, 1768, y en algunos casos como Serpula (Hydroides) norvegica.

Localidades citadas: Cantábrico [Fosa del Capbreton (Golfo de Vizcaya), Guipúzcoa, San Sebastián, Zumaya, Ondarroa, Cabo Santa Catalina, Cabo Villano (citadas en ocasiones de forma genérica como País Vasco), Punta del Pescador, Cabo Mayor, Cierzo y Transval (estos dos últimos citados en ocasiones de forma genérica como Santander), Cabo de Oyambre, Punta Candelaria, Ribadesella, Cabo de Lastres, Gijón, Ribadeo, Foz]; Atlántico [Fornelos (Ría de Ferrol), 
Ría de Ares-Betanzos (en ocasiones citados de forma genérica como Galicia), Islas Sisargas, Cabo Finisterre, Ría de Muros, Ría de Pontevedra, Ensenada de Baiona (Ría de Vigo), costa ibérica de Portugal]; Mediterráneo [Gibraltar, Málaga, Mar Menor, Valencia, Palma de Mallorca, Barcelona, Blanes, Cabo de Creus (citados en ocasiones de forma genérica como Cataluña)].

\section{Hydroides pseudouncinatus pseudouncinatus} Zibrowius, 1971

Referencias: 4, 6, 7, 9, 10, 17, 26, 27, 34, 35, 36, $37,38,39,41,43,85,86,88,116,118,119,125,126$, 130, 141, 145, 147, 149, 150, 151, 157, 159, 169.

Citado como Hydroides uncinata (Philippi, 1844) o Hydroides pseudouncinata pseudouncinata Zibrowius, 1970. Nunca ha sido citado para las costas íbero-baleares con el nombre actualmente válido.

Localidades citadas: Atlántico (A Coruña, costa ibérica de Portugal); Mediterráneo [Gibraltar, Bahía de Algeciras, Mar de Alborán, Málaga, Nerja, Los Escullos, Almería (en ocasiones citadas de forma genérica como Andalucía), Islas Chafarinas, Valencia, Palma de Mallorca, Mahón (estos dos últimos en ocasiones citados de forma genérica como Baleares), Islas Columbretes, Ensenada de los Alfaques, Gerona, Blanes, Cabo de Creus (en ocasiones citados de forma genérica como Cataluña)].

\section{Hydroides stoichadon Zibrowius, 1971 (*)}

Referencias: 143.

Localidades citadas: Cantábrico (Cabo Mayor); Atlántico (Portugal); Mediterráneo (Almería).

Adición al catálogo de Ariño (1987).

Género Janita Saint Joseph, 1894

Janita fimbriata (Delle Chiaje, 1822) (*)

Referencias: 6, 9, 15, $26,27,36,37,39,69,85,86$, $88,118,126,130,147,147,156$.

Citado en algunos casos como Omphalopomopsis fimbriata (Delle Chiaje, 1822), Omphalopoma fimbriata y como Janita spinosa (Langerhans, 1884).

Localidades citadas: Cantábrico (San Sebastián, Santander, Gijón); Mediterráneo [Andalucía, Mar de Alborán, Baleares, Gerona, Cabo de Creus (estos dos últimos citados en ocasiones de forma genérica como Cataluña)].

Género Josephella Caullery \& Mesnil, 1896

Josephella marenzelleri Caullery \& Mesnil, 1896

Referencias: 2, 3, 6, 8, 9, 11, 12, 37, 38, 39, 40, 41, 42, 43, 86, 87, 88, 103, 137, 150, 151, 167.

Localidades citadas: Cantábrico [Guipúzcoa (citado en ocasiones de forma genérica como País Vasco)]; Atlántico [Arrábida (citada en ocasiones de forma genérica como la costa ibérica de Portugal)]; Mediterráneo [Gibraltar, Almería, Islas Chafarinas, Águilas, Murcia, Mar Menor, Valencia, Baleares, Islas Columbretes, Gerona, Cabo de Creus (estos dos últimos citados en ocasiones de forma genérica como Cataluña)].
Género Metavermilia Bush, 1905

Metavermilia multicristata (Philippi, 1844)

Referencias: 6, 9, 15, 16, 26, 27, 29, 36, 37, 39, 69, $137,166,167,170$.

Citado en algunos casos como Vermiliopsis multicristata (Philippi, 1844).

Localidades citadas: Atlántico [Arrábida (citada en ocasiones de forma genérica como la costa ibérica de Portugal)]; Mediterráneo (Andalucía, Mar de Alborán, Cabo de Creus).

\section{Metavermilia taenia Zibrowius 1971}

Referencias: 37, 39, 170.

Localidades citadas: Atlántico (costa ibérica de Portugal).

Género Neovermilia Day, 1961

Neovermilia falcigera (Roule, 1898)

Referencias: 14, 36, 37, 39, 69, 174.

Localidades citadas: Cantábrico (Golfo de Vizcaya); Atlántico (Galicia).

Género Placostegus Philippi, 1844

Placostegus crystallinus sensu Zibrowius, 1968

Referencias: 6, 9, 14, 35, 36, 37, 39, 69, 85, 86, 88 .

Citado como Placostegus crystallinus (Scacchi, 1836).

Localidades citadas: Atlántico (Galicia); Mediterráneo (Baleares, Gerona, Cabo de Creus).

\section{Placostegus langerhansi Marenzeller, 1893}

Referencias: 37, 39, 167.

Localidades citadas: Atlántico (costa ibérica de Portugal).

Placostegus tridentatus (Fabricius, 1779) (*)

Referencias: $15,29,36,37,39,48,69,117,118$, 126, 130, 134, 147, 167.

Citado como Placostegus trydentatus (Fabricius, 1779) o Placostegus tricuspidatus (Sowerby, 1825).

Localidades citadas: Cantábrico [Cierzo y Transval (citados en ocasiones de forma genérica como Santander), Cabo Mayor, Gijón]; Atlántico (costa ibérica de Portugal); Mediterráneo [Mar de Alborán, Valencia, Palma de Mallorca, Mahón (estos dos citados en ocasiones de forma genérica como Baleares), Vinaroz, Cataluña, Golfo de Rosas].

Género Pomatoceros Philippi, 1844

Pomatoceros lamarcki (Quatrefages, 1866) (*)

Referencias: 2, 3, 4, 6, 9, 18, 26, 27, 34, 35, 37, 38, $39,43,85,86,88,94,95,96,101,102,104,106,109$, $126,136,137,138,141,149,150,151,166$.

Localidades citadas: Cantábrico [Golfo de Vizcaya, Guipúzcoa, Sopelana (citados en ocasiones de forma genérica como País Vasco), Cabo Ajo]; Atlántico [Batel, Segaño, Ría de Ferrol, Mugardos, Laxe, Fornelos, Ría de Arousa, Ensenada de Baiona (Ría de Vigo), Arrábida (citado en ocasiones de forma genérica como la costa ibérica de Portugal), Chiclana]; Mediterráneo [Bahía de Algeciras, Gibraltar, Nerja, 
Mar de Alborán, Almería, Ensenada de los Alfaques, Gerona, Islas Medas, Cabo de Creus (citados en ocasiones de forma genérica como Cataluña)].

Pomatoceros triqueter (Linnaeus, 1758) (*)

Referencias: 1, 9, 11, 17, 18, 19, 20, 21, 27, 29, 30, $34,35,37,38,39,41,45,46,50,51,57,59,63,67$, $69,70,74,75,77,78,79,80,84,86,88,93,94,95$, $100,101,104,106,108,110,112,113,116,117,118$, $120,125,126,127,129,130,131,136,137,138,139$, $140,145,146,147,148,150,151,156,157,160,161$, $162,163$.

Localidades citadas: Cantábrico [Guipúzcoa, San Sebastián, Vizcaya (citados en ocasiones de forma genérica como País Vasco), Punta del Pescador, Marona, Transval, Cierzo, Ramo (Santander), San Vicente de la Barquera, Gijón, Playa de la Ribeira (Asturias), Lugo]; Atlántico [Segaño, Ría de Ferrol, Mugardos, Ría de Ares-Betanzos, Fornelos, Batel, Laxe, Ría de Arousa, Pontevedra, Vigo, Islas Cíes, ensenada de Baiona (Ría de Vigo) (citados en ocasiones de forma genérica como Galicia), Arrábida (citado en ocasiones de forma genérica como la costa ibérica de Portugal)]; Mediterráneo [desembocadura del río Piedras (Huelva), Gibraltar, Fuengirola, Almería, Los Escullos, Mar de Alborán, Murcia, Valencia, Baleares, Palma de Mallorca, Islas Columbretes, Barcelona, Gerona, Blanes, Islas Medas (citados en ocasiones de forma genérica como Cataluña)].

Género Protis Ehlers, 1887

Protis arctica (Hansen, 1879)

Referencias: 15, 16, 36, 37, 39, 69.

Localidades citadas: Cantábrico; Atlántico (costa ibérica norte de Portugal).

\section{Género Protula Risso, 1826}

Protula intestinum (Lamarck, 1818)

Referencias: $29,35,36,37,39,58,69,88,118,125$, 126, 130, 135, 150, 151, 166.

Localidades citadas: Mediterráneo [Gibraltar, Mar de Alborán, Valencia, Mahón, Cabrera (estos dos citados en ocasiones de forma genérica como Baleares), Vinaroz, Cataluña, Blanes].

\section{Protula tubularia (Montagu, 1803)}

Referencias: $6,9,15,16,19,29,30,36,37,38,39$, $41,46,48,58,66,69,85,86,88,101,110,114,117$, $118,121,125,126,129,130,130,135,144,147,150$, $151,157,158,166,167$.

Citado en algunos casos como Apomatus similis Marion \& Bobretzky, 1875

Localidades citadas: Cantábrico [Golfo de Vizcaya, Santander, Puerto-Chico (Santander), San Vicente de la Barquera, Gijón]; Atlántico [A Coruña, Ría de Ferrol, Vigo (citados en ocasiones de forma genérica como Galicia), costa ibérica de Portugal]; Mediterráneo [Gibraltar, Málaga, Mar de Alborán, Islas Chafarinas, Murcia, Valencia, Baleares, Mahón, Islas Columbretes, Barcelona, Gerona, Blanes, Golfo de Rosas, Cabo de Creus (citados en ocasiones de forma genérica como Cataluña)].

\section{Género Pseudovermilia Bush, 1907}

Pseudovermilia occidentalis (McIntosh, 1885)

Referencias: 37, 39, 167.

Localidades citadas: Atlántico (costa ibérica de Portugal).

Género Salmacina Claparède, 1870

Salmacina dysteri (Huxley, 1855)

Referencias: $2,3,17,18,19,29,30,36,37,38,39$, $48,69,118,119,121,122,125,126,129,130,134$, 141, 147, 149, 156, 157, 166.

Citado en algunos casos como como Salmacina edificatrix Claparède, 1870, Salmacina aedificatrix Claparède, 1870, y Filograna implexa Berkeley, 1828. Ciertos autores consideran necesaria una revisión detallada del género Salmacina debido a que las diferencias entre las distintas especies que conforman este género son muy sutiles (Bianchi, 1981; Bastida-Zavala, 2009), habiendo realizado comentarios sobre este respecto en Nogueira y Ten Hove (2000). Además, este género puede confundirse con el género Filograna, ya que se diferencian en que los representantes de este último presentan opérculo, de modo que individuos de Filograna que hayan perdido el opérculo pueden ser confundidos fácilmente con ejemplares pertenecientes a Salmacina.

Localidades citadas: Cantábrico [Guipúzcoa (citado en ocasiones de forma genérica como País Vasco), Marona y Cierzo (citados en ocasiones de forma genérica como Santander)]; Atlántico (Vigo, costa ibérica de Portugal); Mediterráneo (Bahía de Algeciras, Gibraltar, Málaga, Nerja, Mar de Alborán, Murcia, Valencia, Islas Columbretes).

Salmacina incrustans Claparède, 1870

Referencias: 26, 27, 29, 36, 37, 38, 39, 42, 44, 54, 69, 70, 119, 125, 126, 130, 142, 146, 166.

Localidades citadas: Cantábrico (País Vasco); Atlántico [A Coruña (citado en ocasiones de forma genérica como Galicia)]; Mediterráneo [Málaga, Almería, Los Escullos (citados en ocasiones de forma genérica como Andalucía), Mar de Alborán, Murcia, Mar Menor, Valencia, Cabrera, Blanes].

Género Semivermilia Ten Hove, 1975

Semivermilia agglutinata (Marenzeller, 1893)

Referencias: 6.

Localidades citadas: Mediterráneo (Cabo de Creus).

Semivermilia crenata (O. G. Costa, 1861)

Referencias: 37,39, 85, 86, 88, 150, 151,171.

Citado en algunos casos como Vermiliopsis crenata (O. G. Costa, 1861).

Localidades citadas: Mediterráneo [Gibraltar, Baleares, Gerona (en ocasiones citado de forma genérica como Cataluña)].

\section{Semivermilia cribrata (O. G. Costa, 1861)}

Referencias: $85,86$. 
Citado en ambas referencias como Semivermilia cribata Zibrowius, 1968.

Localidades citadas: Mediterráneo [Islas Chafarinas, Gerona (en ocasiones citado de forma genérica como Cataluña)].

\section{Semivermilia torulosa (Delle Chiaje, 1822)}

Referencias: 35.

Citado como Vermiliopsis rugosa (Langerhans, 1884).

Localidades citadas: Mediterráneo (Islas Medas).

\section{Género Serpula Linnaeus, 1758}

Serpula cavernicola Fassari \& Mòllica, 1991

Referencia: 175.

Localidades citadas: Atlántico (Sur de Portugal).

Serpula concharum Langerhans, 1880 (*)

Referencias: 2, 3, 4, 6, 7, 9, 10, 11, 14, 15, 17, 26, $27,29,34,35,36,37,38,39,42,43,55,69,85,86$, $88,101,114,116,118,121,126,129,130,137,138$, 141, 147, 149, 150, 151, 156, 157, 166, 167.

Localidades citadas: Cantábrico [Fosa del Capbreton (Golfo de Vizcaya), Guipúzcoa, San Sebastián, Zumaya, Cabo Santa Catalina, (en ocasiones citados de forma genérica como País Vasco), Cabo Mayor, Cierzo y Ramo (en ocasiones citados de forma genérica como Santander), Santander, Cabo de Oyambre, Gijón, Cabo de Peñas, El Cachucho, Ribadeo, Foz]; Atlántico [A Coruña, Ría de Ferrol, Fornelos, Islas Sisargas (en ocasiones citados de forma genérica como Galicia), Arrábida (citado en ocasiones como la costa ibérica de Portugal)]; Mediterráneo [Bahía de Algeciras, Gibraltar, Nerja, Almería, Mar de Alborán, Islas Chafarinas, Mar Menor, Valencia, Baleares, Islas Columbretes, Gerona, Islas Medas, Blanes, Cabo de Creus (en ocasiones citados de forma genérica como Cataluña)].

\section{Serpula lobiancoi Rioja, 1917 (*)}

Referencias: 26, 27, 29, 30, 31, 35, 36, 37, 39, 69, $85,86,88,116,118,121,123,126,130,147,150$, $151,156,166,167$.

Localidades citadas: Cantábrico (Cabo de Oyambre, Gijón); Atlántico (costa ibérica de Portugal); Mediterráneo [Gibraltar, Andalucía, Mar de Alborán, Valencia, Gerona (citado en ocasiones de forma genérica como Cataluña)].

\section{Serpula vermicularis Linnaeus, 1767 (*)}

Referencias: 4, 6, 7, 9, 10,11, 14, 15, 17, 26, 27, 29, $31,33,34,35,36,37,38,39,41,42,43,45,46,48$, $55,61,65,69,70,72,75,77,85,86,88,89,94,97$, $98,101,104,106,110,115,116,117,118,119,120$, $121,125,126,127,129,130,131,132,134,135,137$, $138,140,141,146,147,149,150,151,153,156,157$, $158,166,167$.

Citado en algunos casos como Serpula aspera Philippi, 1844.

Localidades citadas: Cantábrico [Fosa del Capbreton (Golfo de Vizcaya), País Vasco, San Sebastián,
Cabo Villano, Laredo, Cabo Ajo, Santander, Cabo Mayor, San Vicente de la Barquera, Punta Candelaria, Ribadesella, Gijón, Cabo de Peñas, El Cachucho]; Atlántico [Ría de Ferrol, Mugardos, Ría de Coruña, Banco de Galicia, Cabo Finisterre, Ría de Arousa, Ría de Pontevedra, Marín, Ensenada de Baiona (Ría de Vigo), Vigo (citados en ocasiones de forma genérica como Galicia), Arrábida (citado en ocasiones de forma genérica como la costa ibérica de Portugal)]; Mediterráneo [desembocadura del río Piedras (Huelva), Bahía de Algeciras, Gibraltar, Mar de Alborán, Málaga, Fuengirola, Nerja, Islas Chafarinas, Almería, Los Escullos, Murcia, Mar Menor, Valencia, Baleares, Punta de San Carlos (Palma de Mallorca), Islas Columbretes, Barcelona, Gerona, Blanes, Islas Medas, Cabo de Creus].

Género Spiraserpula Regenhardt, 1961

Spiraserpula massiliensis (Zibrowius, 1968)

Referencias: 4, 34, 35, 37, 39, 60, 85, 86, 88, 141.

Citado en la mayoría de referencias como Serpula massiliensis Zibrowius, 1968.

Localidades citadas: Mediterráneo [Nerja, Almería, Baleares, Gerona (citado en ocasiones de forma genérica como Cataluña)].

\section{Género Spirobranchus Blainville, 1818}

Spirobranchus lima (Grube, 1862)

Referencias: $35,116$.

Localidades citadas: Mediterráneo (Valencia, Islas Medas).

Adición al catálogo de Ariño (1987).

\section{Spirobranchus polytrema (Philippi, 1844)}

Referencias: 2, 3, 4, 6, 7, 8, 9, 10, 11, 12, 13, 26, 27, $27,29,34,35,36,37,38,39,40,41,48,57,67,69$, $70,85,86,88,93,116,117,118,122,123,126,129$, $130,137,138,139,141,142,145,146,147,148,149$, 149, 150, 151, 156, 157.

Citado en algunos casos como Pomatostegus polytrema (Philippi, 1844).

Localidades citadas: Cantábrico [Guipúzcoa (citado en ocasiones de forma genérica como País Vasco), Santander, San Vicente de la Barquera, Lugo]; Atlántico [Arrábida (citada en ocasiones de forma genérica como la costa ibérica de Portugal)]; Mediterráneo [Bahía de Algeciras, Gibraltar, Nerja, Almería, Los Escullos, Mar de Alborán, Islas Chafarinas, Águilas (citado en ocasiones de forma genérica como Murcia), Valencia, Palma de Mallorca, Cala Ganduf y L'Olla (Cabrera) (citados en ocasiones de forma genérica como Baleares), Gerona, Islas Medas, Cabo de Creus (citado en ocasiones de forma genérica como Cataluña)].

\section{Género Vermiliopsis Saint-Joseph, 1894 \\ Vermiliopsis infundibulum (Philippi, 1844) $(*)$}

Referencias: $4,6,7,9,10,14,15,16,17,18,26,27$, $29,34,35,36,37,38,39,41,42,44,69,85,86,88$, $114,116,117,118,119,125,126,129,130,131,137$, $138,141,147,150,151,156,157,159,166$. 
Localidades citadas: Cantábrico [Golfo de Vizcaya, Cierzo y Transval (Santander), Cabo Mayor, Santander, Cabo de Oyambre, Gijón]; Atlántico [A Coruña (citado en ocasiones de forma genérica como Galicia), Islas Sisargas, Arrábida (citado en ocasiones de forma genérica como la costa ibérica de Portugal)]; Mediterráneo [Gibraltar, Nerja, Málaga, Mar de Alborán, Islas Chafarinas, Mar Menor, Valencia, Palma de Mallorca, Mahón (citados en ocasiones de forma genérica como Baleares), Islas Columbretes, Vinaroz, Gerona, Blanes, Costa Brava, Cabo de Creus (citados en ocasiones de forma genérica como Cataluña)].

Vermiliopsis labiata (O. G. Costa, 1861)

Referencias: 4, 6, 9, 27, 35, 37, 38, 39, 85, 86, 88, 116, 141, 154, 156.

Citado en algunos casos como Vermiliopsis richardi Fauvel, 1909.

Localidades citadas: Mediterráneo (Gibraltar, Nerja, Mar de Alborán, Valencia, Baleares, Islas Columbretes, Gerona, Cabo de Creus).

Vermiliopsis monodiscus Zibrowius, 1968 (*)

Referencias: 26, 27, 28, 88, 156.

Localidades citadas: Atlántico [Banco de Galicia (primera cita para las costas atlánticas ibéricas)]; Mediterráneo (Andalucía, Mar de Alborán, Baleares).

Vermiliopsis striaticeps (Grube, 1862)

Referencias: 4, 6, 9, 26, 27, 44, 85, 86, 88, 94, 95, 101, 104, 107, 116, 141, 143, 150, 157, 158.

Citado en algunos casos como Vermilia striaticeps (Grube, 1862).

Localidades citadas: Atlántico [Ría de Ferrol, A Redonda, ensenada de Baiona (Ría de Vigo)]; Mediterráneo [Nerja, Almería, Los Escullos, Mar de Alborán, Islas Chafarinas, Mar Menor, Valencia, Baleares, Gerona, Cabo de Creus (en ocasiones citados de forma genérica como Cataluña)].

Subfamilia Spirorbinae Chamberlin, 1919

Género Circeis Saint-Joseph, 1894

Circeis armoricana Saint-Joseph, 1894

Referencias: 36, 37, 39, 69, 129, 130.

Citado en algunos casos como Spirorbis (Dexiospira) armoricanus, Spirorbis armoricanus, y Dexiospira armoricana.

Localidades citadas: Cantábrico (Santander).

Género Janua Saint-Joseph, 1894

Janua pagenstecheri (Quatrefages, 1866)

Referencias: 6, 9, 13, 17, 18, 36, 37, 38, 39, 69, 81, $82,88,96,101,118,119,121,122,125,126,127,129$, 130, 137, 138, 147, 147, 148, 150, 151, 157, 158, 166.

Citado en algunos casos como Spirorbis (Dexiospira) pagenstecheri, Dexiospira pagenstecheri, у Janua (Janua) pagenstecheri.

Localidades citadas: Cantábrico [Guipúzcoa, Sopelana, Sables y Magdalena (Santander), Gijón]; Atlántico [Ría de Ferrol, Fornelos, Laxe, Mugardos, Batel,
A Coruña, Ría de Arousa, Ría de Vigo, Islas Cíes (citados en ocasiones de forma genérica como Galicia), Arrábida (citado en ocasiones como la costa ibérica de Portugal)]; Mediterráneo (Gibraltar, Málaga, Mar de Alborán, Islas Chafarinas, Águilas, Valencia, Baleares, Gerona, Blanes, Islas Medas, Cabo de Creus).

Género Laeospira Caullery \& Mesnil, 1897

Laeospira corallinae (de Silva \& Knight-Jones, 1962)

Referencias: 101, 105.

Citado como Spirorbis corallinae de Silva \& Knight-Jones, 1962.

Localidades citadas: Atlántico (Segaño, Ría de Ferrol, Laxe, Batel).

Adición al catálogo de Ariño (1987). Especie conocida en las costas británicas e irlandesas (Parapar, 1991).

Género Neodexiospira Pillai, 1970

Neodexiospira pseudocorrugata (Bush, 1905)

Referencias: $6,8,9,11,29,37,39,64,69,73,88$, 126, 127, 129, 130, 137, 150, 151, 157, 158, 166.

Citado como Janua pseudocorrugata (Bush, 1905).

Localidades citadas: Mediterráneo (Gibraltar, Mar de Alborán, Islas Chafarinas, Baleares, Gerona, Cabo de Creus).

Género Paradexiospira Caullery \& Mesnil, 1897

Paradexiospira vitrea (O. Fabricius, 1780)

Referencias: 13, 148.

Citado como Spirorbis vitreus (Fabricius, 1780).

Localidades citadas: Cantábrico (Guipúzcoa, San Sebastián).

Género Paralaeospira Caullery \& Mesnil, 1897

Paralaeospira malardi Caullery \& Mesnil, 1897

Referencias: 36, 37, 39, 69, 69, 126, 130.

Citado en algunos casos como Spirorbis

(Paralaeospira) malardi Caullery \& Mesnil, 1897.

Localidades citadas: Cantábrico (Santander).

Género Pileolaria Claparède, 1868

Pileolaria heteropoma (Zibrowius, 1968)

Referencias: 88

Localidades citadas: Mediterráneo (Baleares).

Pileolaria militaris Claparède, 1870

Referencias: 6, 8, 9, 11, 36, 37, 38, 39, 64, 67, 69, $85,86,88,101,119,125,126,127,130,137,147$, $150,151,157,158,166$.

Citado en algunos casos como Spirorbis cornuarietis, Spirorbis militaris (Claparède, 1870), Spirorbis (Laeospira) cornu-arietis, Spirorbis (Laeospira) militaris, y Spirorbis mediterraneus (Caullery \& Mesnil, 1897).

Localidades citadas: Atlántico [Ría de Ferrol, A Coruña, Fornelos, Batel (en ocasiones citados de forma genérica como Galicia), Arrábida]; Mediterráneo [Gibraltar, Málaga, Mar de Alborán, Islas Chafarinas, Águilas, Valencia, Baleares, Mahón, Gerona, Islas 
Medas, Cabo de Creus (en ocasiones citados de forma genérica como Cataluña)].

Género Protolaeospira Pixell, 1912

Protolaeospira striata (Quiévreux, 1963)

Referencias: 37, 39, 85, 86, 88, 137.

Citado bien como Protalospira striata o como

Protolaeospira striata (Quiévreux, 1963).

Localidades citadas: Mediterráneo [Islas Chafarinas, Baleares, Gerona (en ocasiones citado de forma genérica como Cataluña)].

Género Spirorbis Daudin, 1800

Spirorbis corrugatus (Montagu, 1803)

Referencias: 29, 36, 37, 69, 126, 127, 129, 130, 137, 139, 142, 145, 149.

Citado en ocasiones como Spirorbis (Dexiospira) corrugatus (Montagu, 1803), o bien Dexiospira corrugata (Montagu, 1803).

Localidades citadas: Cantábrico (Santander, San Vicente de la Barquera, Gijón); Atlántico [A Coruña, Pontevedra, Marín (estas tres localidades citadas en ocasiones de forma genérica como Galicia), Arrábida]; Mediterráneo (Bahía de Algeciras, Palma de Mallora, Cabrera).

\section{Spirorbis cuneatus Gee, 1964}

Referencias: 37, 39, 88, 98, 99, 101, 137, 150, 151.

Citado en ocasiones como Spirorbis communis Bosc, 1802, o bien como Spirorbis cuneatus Gee, 1964.

Localidades citadas: Atlántico [Ría de Ferrol, Batel, Arrábida (citado en ocasiones de forma genérica como Portugal)]; Mediterráneo (Gibraltar).

Spirorbis infundibulum Harris \& Knight-Jones, 1964 Referencias: 35, 36, 37, 64, 69, 137.

Citado en ocasiones como Spirorbis (Laeospira) infundibulum Harris \& Knight-Jones, 1964, Spirorbis striatus Knight-Jones, 1978, Paralaeospira striatus o principalmente como Spirorbis infundibulum Harris \& Knight-Jones, 1964.

Localidades citadas: Atlántico (Arrábida); Mediterráneo (Gerona).

Spirorbis inornatus L'Hardy \& Quièvreux, 1962

Referencias: 101, 105.

Localidades citadas: Atlántico (Ría de Ferrol, Fornelos, Laxe, Batel).

Adición al catálogo de Ariño (1987). Especie conocida en las costas británicas e irlandesas (Parapar, 1991).

Spirorbis marioni Caullery \& Mesnil, 1897

Referencias: 32, 165, 172, 173.

Citado como Spirorbis marioni Caullery \& Mesnil, 1897.

Localidades citadas: Mediterráneo [Tarifa, Puerto de la Selva (Gerona)].

Spirorbis spirorbis (Linnaeus, 1758)

Referencias: $36,37,39,69,101,105,117,118,121$, 126, 127, 129, 130, 130, 147.
Citado en ocasiones como Spirorbis borealis Daudin, 1800 , o bien como Spirorbis spirorbis (Linnaeus, 1758).

Localidades citadas: Cantábrico [Magdalena, San Martín, Sardinero, Isla de Santa Marina, Isla de la Torre, Peña Vieja (localidades de Santander), San Vicente de la Barquera, Gijón]; Atlántico (Ría de Ferrol, Mugardos, Fornelos, Batel).

\section{Spirorbis tridentatus Levinsen, 1883}

Referencias: 39, 73, 88, 105, 126, 130.

Citado como Spirorbis laevis Quatrefages, 1866, o principalmente como Spirorbis tridentatus Levinsen, 1883.

Localidades citadas: Mediterráneo (Baleares).

\section{Discusión}

\section{DISTRIBUCIÓN Y ESPECIES ALÓCTONAS}

El presente catálogo incrementa en 4 el número de especies de serpúlidos íbero-baleares respecto al de Ariño (1987), aunque cabe destacar que en otras 20 especies se han producido cambios en la nomenclatura desde entonces. Las 4 especies que no aparecían en el catálogo de Ariño (1987) y que se incluyen como adiciones al mismo, se citan a partir de literatura más actualizada: Spirobranchus lima (de distribución mediterránea como se especifica en este catálogo; Camp \& Viñolas, 1984), Spirorbis inornatus y Laeospira corallinae (citadas anteriormente sólo en las costas británicas e irlandesas, como se puede consultar en este catálogo; ambas especies citadas por Parapar, 1991) y la especie Hydroides stoichadon, de ámbito mediterráneo, ampliamente distribuida por las costas italianas y del sureste francés (Ten Hove \& Kupriyanova, 2009). Esta última fue citada por primera vez en Almería (San Martín et al., 1990) e identificada en este estudio a partir de muestras recogidas en Cabo Mayor (Cantabria, Fauna II), siendo ésta la primera cita de esta especie para las costas cantábricas. El hecho de que a H. stoichadon se le atribuyese una distribución únicamente mediterránea y que haya sido encontrada en el Cantábrico podría deberse simplemente a un mayor esfuerzo de estudio en dichas costas.

\section{Quimeras BIOPERCULADAS}

Los registros de serpúlidos que presentaban la anomalía denominada quimera bioperculada fueron revisados por Ten Hove \& Ben-Eliahu (2005). En dicho estudio se demostró cómo en trabajos anteriores se clasificaron erróneamente ejemplares con esta anomalía como pertenecientes a la especie Hydroides norvegicus, por ser muy similares a $H$. elegans. Los ejemplares de la campaña Fauna II serían por tanto los primeros casos documentados de este fenómeno en nuestra región para la especie H. norvegicus (Fig. 1). 
Fue Okada (1933) uno de los primeros en trabajar con individuos bioperculados de Hydroides norvegicus, estudiando el fenómeno que denominó "regulación compensatoria". Este mecanismo fue documentado en Hydroides dianthus e H. norvegicus, $\mathrm{y}$ consiste en que, tras extirparles experimentalmente distintas porciones del opérculo a individuos de estas dos especies, el pseudopérculo comenzaba a desarrollarse hasta adquirir el tamaño y forma del opérculo típico de cada especie. En el caso de H. norvegicus vio que esto sucedía tan sólo retirando el verticilo opercular de espinas (tipo Hydroides) y se dejaba intacto el verticilo basal, tipo Serpula. Esta puede ser una de las posibles explicaciones para las quimeras bioperculadas encontradas en los lotes analizados: un ejemplar de $H$. norvegicus que ha perdido el verticilo superior del opérculo (por predación o cualquier otra razón) y cuyo pseudopérculo se ha desarrollado como opérculo maduro, quedándose un individuo bioperculado, con el opérculo original con aspecto de opérculo tipo Serpula.

La Greca (1949), llegó a conclusiones similares realizando su estudio en condiciones naturales. Defendió que los individuos bioperculados que aparecían en dichas condiciones naturales no eran malformaciones, sino una condición filogenéticamente primitiva que aparece como un fenómeno de regeneración ante daño opercular mediado por la represión del desarrollo que ejerce el opérculo sobre el pseudopérculo.

Si bien esta teoría de Okada (1933) y La Greca (1949) parece en principio una explicación plausible para los individuos que hemos descrito a partir de las muestras de Fauna II, no se encontraron roturas evidentes en la parte superior del opérculo tipo Serpula, por lo que nos inclinamos a pensar que se trata de un estado ontogenético transitorio y muy breve, como lo definieron Ten Hove \& Ben-Eliahu (2005). Estos autores defendieron así la existencia de un nuevo estado opercular, existiendo por lo tanto tres: pseudopérculo, opérculo tipo priscus (antes nombrado como tipo Serpula) y opérculo maduro.

Las quimeras bioperculadas de especies del género Hydroides que estudiaron estos autores tienen un origen biogeográfico común, ya que provienen todas del Indo-Pacífico Oeste, con contadas excepciones de migrantes lessepsianos. Aunque Hydroides norvegicus fue descrito por primera vez en Noruega, se piensa que su origen se encuentra en el Atlántico Oriental y la zona Indo-Pacífica (Pillai, 1972), y que pudo ser una de las especies que se adherían a los cascos de los barcos de largo recorrido de hace cientos de años y llegar a las costas atlánticas y mediterráneas de Europa, no como migrante lessepsiano, sino como serpúlido del fouling. Por tanto, se encontraría entre esas especies bioperculadas de Hydroides provenientes de esa región del globo.

Por todo lo que hemos visto, resulta de especial interés destacar la necesidad de profundizar los estudios respecto a esta familia, a nivel regional y global, ya que hay ciertos aspectos que requieren de una importante revisión, como la clasificación taxonómica a nivel de subfamilias y complejos de géneros, así como la importancia de estudios en cuanto a la relación entre esta familia y el fouling, actividad de especial interés económico, ya que se desconocen los efectos que pueden provocar las especies de serpúlidos alóctonos introducidos en nuestra región.

\section{Agradecimientos}

Agradecemos al personal del Museo Nacional de Ciencias Naturales de Madrid, especialmente a Javier Sánchez Almazán, María Ángeles Ramos y Manuel Sánchez el préstamo del material utilizado para la realización del trabajo, tanto las muestras biológicas como la información acerca de la campaña Fauna II. Asimismo agradecemos a la Dra. Marta Pola (Departamento de Biología, Zoología, Facultad de Ciencias, Universidad Autónoma de Madrid) el préstamo de la lupa Nikon SMZ-1500 para la realización de la fotografía presentada. También queremos agradecer a dos anónimos revisores sus sugerencias que han contribuido a la mejora de este artículo.

\section{Referencias}

Ariño, A. H., 1987. Bibliografía Ibérica de Poliquetos. Base de datos y Catálogo de especies. Publicaciones de Biología de la Universidad de Navarra, Serie Zoológica, 16: 1-176.

Bastida-Zavala, J. R., 2009. Capítulo 43: Serpulidae Rafinesque, 1815. In: de León-González, J. A., BastidaZavala, J. R., Carrera-Parra, L. F., García-Garza, M. E., Peña-Rivera, A., Salazar-Vallejo, S. I. \& Solís-Weiss, V. (Eds.) Poliquetos (Annelida: Polychaeta) de México y América Tropical. Universidad Autónoma de Nuevo León. Monterrey: 521-554.

Bianchi, C. N., 1981. Policheti Serpuloidei. Guide per il riconoscimento delle specie animali delle acque lagunari e costiere italiane, 5: 1-87.

Camp, J. \& Viñolas, C., 1984. Capítulo 25: Els Poliquets de les Illes Medes. In: Ros, J., Olivella, I. \& Gili, J. M. (eds.) Els Sistemes naturals de les illes Medes. Institut d'Estudis Catalans. Barcelona: 443-455.

Campoy, A., 1982. Fauna de España. Fauna de Anélidos poliquetos de la Península Ibérica (II). Publicaciones de Biología de la Universidad de Navarra, Serie Zoológica, 7: 1-781.

Emara, A. M. \& Belal, A. A., 2004. Marine fouling in Suez Canal, Egypt. Egyptian Journal of Aquatic Research, 30(A): 189-206.

Fauchald, K., 1977. The Polychaete Worms. Definition and Keys to the Orders, Families and Genera. Natural History Museum of Los Angeles County, Science Series, 28: 1-188.

Fitzhugh, K., 1989. A Systematic Revision of the Sabellidae-Caobangiidae-Sabellongidae Complex (Annelida: Polychaeta). Bulletin of the American Museum of Natural History, 192: 1-104.

Kupriyanova, E. K. \& Rouse, G. W., 2008. Yet another example of paraphyly in Annelida: Molecular evidence that Sabellidae contains Serpulidae. Molecular 
Phylogenetics and Evolution, 46: 1174-1181. http://dx. doi.org/10.1016/j.ympev.2007.10.025

Gil, J. C. F., 2011. The European Fauna of Annelida Polychaeta (II). Tesis Doctoral. Facultad de Ciencias, Departamento de Biología Animal. Universidade de Lisboa. $1554 \mathrm{pp}$.

La Greca, M., 1949. Richerche sull'origine degli individui biopercolati di Hydroides (Anellidi Policheti). Bolletino di Zoologia, 16: 4-6.

Lehrke, J., Ten Hove, H. A., MacDonald, T. A., Bartolomaeus, T. \& Bleidorn, C., 2007. Phylogenetic relationships of Serpulidae (Annelida: Polychaeta) based on $18 \mathrm{~S}$ rDNA sequence data, and implications for opercular evolution. Organisms, Diversity \& Evolution, 7: 195-206. http://dx.doi.org/10.1016/j.ode.2006.06.004

Nogueira, J. M. de Matos \& Ten Hove, H. A., 2000. On a new species of Salmacina Claparède, 1870 (Polychaeta: Serpulidae) from Sao Paolo State, Brazil. Beaufortia, 50: $151-161$

Okada, Y. K., 1933. Remarks on the Reversible Asymmetry in the Opercula of the Polychaete Hydroides. Journal of the Marine Biological Association of the United Kingdom (New Series), 18: 655-670. http://dx.doi. org/10.1017/S0025315400043976

Parapar, J., 1991. Anélidos Poliquetos bentónicos de la Ría de Ferrol (Galicia). Tesis Doctoral. Universidad de Santiago de Compostela. 1104 pp.

Pillai, T. G., 1970. Studies on a collection of spirorbids from Ceylon, together with a critical review and revision of spirorbid systematic and an account of their phylogeny and zoogeography. Ceylon Journal of Science (Biological Sciences), 8: 100-172.

Pillai, T. G., 1972. A Review and Revision of the Systematics of the Genera Hydroides and Eupomatus together with an Account on their Phylogeny and Zoogeography. Ceylon Journal of Science (Biological Sciences), 10(1): $1-25$.

Rouse, G. W., 2001. Capítulo 50: Serpulidae Latreille, 1825. In: Rouse, G. W. \& Pleijel, F. (Eds.) Polychaetes. Oxford University Press. Hong Kong: 198-201.

San Martín, G., Estapé, S., García-Ocejo, A., Gómez, C. \& Jiménez, P., 1990. Estudio de la taxocenosis de anélidos poliquetos de rizomas de Posidonia oceanica en las costas de Almería. Boletín del Instituto Español de Oceanografía, 6(1): 41-58.

Ten Hove, H. A. \& Ben-Eliahu, M. N., 2005. On the identity of Hydroides priscus Pillai 1971-Taxonomic confusion due to ontogeny in some serpulid genera (Annelida, Polychaeta: Serpulidae). Senckenbergiana biologica, 85(2): 1-19.

Ten Hove, H. A. \& Kupriyanova, E. K., 2009. Taxonomy of Serpulidae (Annelida, Polychaeta): The state of affairs. Zootaxa, 2036: 1-126.

WoRMS Editorial Board, 2016. World Register of Marine Species. Disponible en http://www.marinespecies.org. Vlaams Instituut voor de Zee.

Zibrowius, H., 1968. Etude morphologique, systématique et écologique des Serpulidae (Annelida: Polychaeta) de la région de Marseille. Recueil des Travaux de la Station Marine d'Endoume, 45(59): 81-252. 
Apéndice 1.-Estaciones de la campaña oceanográfica Fauna // del proyecto "Fauna lbérica" (1991) en las que se colectaron ejemplares de la Familia Serpulidae identificados en este estudio.

Appendix 1.-Stations of the oceanographic cruise Fauna // of the Project "Fauna Ibérica" (1991) in which the specimens of the Family Serpulidae identified in this study were collected.

\begin{tabular}{|c|c|c|c|c|}
\hline ESTACIÓN & LOCALIDAD & PROVINCIA & COORDENADAS & PROF. (m) \\
\hline $85 \mathrm{DH}$ & Ría de Muros & A Coruña & $42^{\circ} 41.57^{\prime}-42^{\circ} 41.72^{\prime} \mathrm{N}-9^{\circ} 10.17^{\prime}-9^{\circ} 10.45^{\prime} \mathrm{O}$ & $85-85$ \\
\hline $91 \mathrm{~A}$ & Cabo Finisterre & A Coruña & $42^{\circ} 52.85^{\prime}-42^{\circ} 50.93^{\prime} N-9^{\circ} 21.47^{\prime}-9^{\circ} 20.91^{\prime} \mathrm{O}$ & $129-133$ \\
\hline $98 \mathrm{~A}$ & Islas Sisargas & A Coruña & $43^{\circ} 25.73^{\prime}-43^{\circ} 26.34^{\prime} N-8^{\circ} 59.76^{\prime}-8^{\circ} 58.95^{\prime} O$ & $157-159$ \\
\hline $101 \mathrm{~A}$ & Coruña & A Coruña & $43^{\circ} 35.58^{\prime}-43^{\circ} 35.53^{\prime} N-8^{\circ} 34.28^{\prime}-8^{\circ} 31.99^{\prime} O$ & $151-152$ \\
\hline $104 \mathrm{P}$ & Coruña & A Coruña & $44^{\circ} 14.01^{\prime}-44^{\circ} 13.82^{\prime} N-8^{\circ} 37.50^{\prime}-8^{\circ} 34.74^{\prime} O$ & 60 \\
\hline $105 \mathrm{~A}$ & Foz & Lugo & $43^{\circ} 58.75^{\prime}-43^{\circ} 58.18^{\prime} N-7^{\circ} 13.12^{\prime}-7^{\circ} 10.48^{\prime} O$ & $174-180$ \\
\hline $106 \mathrm{~A}$ & Foz & Lugo & $43^{\circ} 55.81^{\prime}-43^{\circ} 53.91^{\prime} N-7^{\circ} 09.01^{\prime}-7^{\circ} 07.74^{\prime} \mathrm{O}$ & $158-167$ \\
\hline $108 \mathrm{~A}$ & Ribadeo & Lugo & $43^{\circ} 42.51^{\prime}-43^{\circ} 42.82^{\prime} N-6^{\circ} 59.30^{\prime}-7^{\circ} 01.10^{\prime} \mathrm{O}$ & 125 \\
\hline $109 \mathrm{~A}$ & Ribadeo & Lugo & $43^{\circ} 39.04^{\prime}-43^{\circ} 50.83^{\prime} N-6^{\circ} 59.38^{\prime}-6^{\circ} 59.58^{\prime} O$ & $142-145$ \\
\hline $111 \mathrm{~A}$ & Cabo de Peñas & Asturias & $43^{\circ} 47.72^{\prime}-43^{\circ} 48.60^{\prime} N-6^{\circ} 05.96^{\prime}-6^{\circ} 04.20^{\prime} O$ & $176-200$ \\
\hline $112 \mathrm{DH}$ & Cabo de Peñas & Asturias & $43^{\circ} 43.72^{\prime}-43^{\circ} 43.82^{\prime} N-5^{\circ} 56.97^{\prime}-5^{\circ} 56.85^{\prime} \mathrm{O}$ & $122-129$ \\
\hline $113 \mathrm{~A}$ & Gijón & Asturias & $43^{\circ} 43.57^{\prime}-43^{\circ} 43.85^{\prime} N-5^{\circ} 55.67^{\prime}-5^{\circ} 56.76^{\prime} O$ & $116-120$ \\
\hline $114 \mathrm{~A}$ & Gijón & Asturias & $43^{\circ} 37.71^{\prime}-43^{\circ} 38.36^{\prime} N-5^{\circ} 37.59^{\prime}-5^{\circ} 39.78^{\prime} O$ & $72-74$ \\
\hline $123 \mathrm{~A}$ & Ribadesella & Asturias & $43^{\circ} 38.43^{\prime}-43^{\circ} 39.38^{\prime} N-5^{\circ} 4.67^{\prime}-5^{\circ} 5.48^{\prime} O$ & $151-152$ \\
\hline $124 \mathrm{~A}$ & Ribadesella & Asturias & $43^{\circ} 49.76^{\prime}-43^{\circ} 49.89^{\prime} N-5^{\circ} 05.04^{\prime}-5^{\circ} 05.83^{\prime} O$ & $540-543$ \\
\hline $128 \mathrm{~A}$ & Cabo Mayor & Cantabria & $43^{\circ} 35.92^{\prime}-43^{\circ} 35.63^{\prime} N-3^{\circ} 47.53^{\prime}-3^{\circ} 49.21^{\prime} O$ & $141-142$ \\
\hline $131 \mathrm{~A}$ & Cabo Ajo & Santander & $43^{\circ} 30.85^{\prime}-43^{\circ} 30.86^{\prime} N-3^{\circ} 40.07^{\prime}-3^{\circ} 40.08^{\prime} O$ & 41 \\
\hline $134 \mathrm{~A}$ & Cabo Mayor & Santander & $43^{\circ} 30.91^{\prime}-43^{\circ} 30.83^{\prime} N-3^{\circ} 47.26^{\prime}-3^{\circ} 46.13^{\prime} O$ & $39-40$ \\
\hline $141 \mathrm{~A}$ & Punta del Pescador & Santander & $43^{\circ} 27.21^{\prime}-43^{\circ} 27.90^{\prime} N-3^{\circ} 23.94^{\prime}-3^{\circ} 23.99^{\prime} O$ & 23-32 \\
\hline $142 \mathrm{~A}$ & Punta del Pescador & Santander & $43^{\circ} 28.04^{\prime}-43^{\circ} 28.91^{\prime} \mathrm{N}-3^{\circ} 24.16^{\prime}-3^{\circ} 24.40^{\prime} \mathrm{O}$ & $36-46$ \\
\hline $143 \mathrm{~A}$ & Punta del Pescador & Santander & $43^{\circ} 29.91^{\prime}-43^{\circ} 30.49^{\prime} \mathrm{N}-3^{\circ} 24.45^{\prime}-3^{\circ} 24.50^{\prime} \mathrm{O}$ & $57-68$ \\
\hline $145 \mathrm{~A}$ & Cabo Villano & Vizcaya & $43^{\circ} 28.62^{\prime}-43^{\circ} 28.64^{\prime} N-2^{\circ} 53.77^{\prime}-2^{\circ} 53.36^{\prime} O$ & 88 \\
\hline $148 \mathrm{~A}$ & San Sebastián & Guipúzcoa & $43^{\circ} 29.56^{\prime}-43^{\circ} 29.88^{\prime} \mathrm{N}-2^{\circ} 01.58^{\prime}-2^{\circ} 00.20^{\prime} \mathrm{O}$ & $135-143$ \\
\hline $150 \mathrm{~A}$ & Zumaya & Guipúzcoa & $43^{\circ} 23.18^{\prime}-43^{\circ} 23.14^{\prime} \mathrm{N}-2^{\circ} 15.00^{\prime}-2^{\circ} 15.21^{\prime} \mathrm{O}$ & $93-101$ \\
\hline $151 \mathrm{~A}$ & Cabo Santa Catalina & Guipúzcoa & $43^{\circ} 25.29^{\prime}-43^{\circ} 25.09^{\prime} N-2^{\circ} 31.05^{\prime}-2^{\circ} 29.83^{\prime} O$ & $82-86$ \\
\hline $152 \mathrm{~A}$ & San Sebastián & Guipúzcoa & $43^{\circ} 22.59^{\prime}-43^{\circ} 22.16^{\prime} \mathrm{N}-2^{\circ} 3.70^{\prime}-2^{\circ} 5.76^{\prime} \mathrm{O}$ & 104-107 \\
\hline $153 \mathrm{~A}$ & Ondarroa & Vizcaya & $43^{\circ} 27.24^{\prime}-43^{\circ} 27.77^{\prime} N-2^{\circ} 23.42^{\prime}-2^{\circ} 24.81^{\prime} \mathrm{O}$ & $129-132$ \\
\hline $156 \mathrm{~A}$ & Cabo de Oyambre & Cantabria & $43^{\circ} 25.37^{\prime}-43^{\circ} 26.06^{\prime} \mathrm{N}-4^{\circ} 11.33^{\prime}-4^{\circ} 08.59^{\prime} \mathrm{O}$ & $39-40$ \\
\hline $158 \mathrm{~A}$ & Cabo de Oyambre & Santander & $43^{\circ} 29.43^{\prime}-43^{\circ} 29.41^{\prime} N-4^{\circ} 20.89^{\prime}-4^{\circ} 20.68^{\prime} O$ & $136-137$ \\
\hline $162 \mathrm{~A}$ & "El Cachucho" & Asturias & $44^{\circ} 02.24^{\prime}-44^{\circ} 02.31^{\prime} \mathrm{N}-4^{\circ} 50.47^{\prime} 4^{\circ} 51.09^{\prime} \mathrm{O}$ & $580-600$ \\
\hline $163 \mathrm{~A}$ & "El Cachucho" & Asturias & $44^{\circ} 02.53^{\prime}-44^{\circ} 2.59^{\prime} \mathrm{N}-4^{\circ} 51.89^{\prime}-4^{\circ} 54.00^{\prime} \mathrm{O}$ & $504-509$ \\
\hline $164 \mathrm{~A}$ & Cabo de Lastres & Asturias & $43^{\circ} 40.27^{\prime}-43^{\circ} 40.06^{\prime} N-5^{\circ} 13.36^{\prime}-5^{\circ} 14.35^{\prime} \mathrm{O}$ & 146 \\
\hline $168 \mathrm{~A}$ & Punta Candelaria & A Coruña & $43^{\circ} 45.13^{\prime}-43^{\circ} 46.53^{\prime} \mathrm{N}-8^{\circ} 10.09^{\prime}-8^{\circ} 9.59^{\prime} \mathrm{O}$ & $116-120$ \\
\hline $170 \mathrm{~A}$ & Islas Sisargas & A Coruña & $43^{\circ} 36.81^{\prime}-43^{\circ} 35.77^{\prime} N-8^{\circ} 44.37^{\prime}-8^{\circ} 43.19^{\prime} O$ & $165-174$ \\
\hline $171 \mathrm{~A}$ & Islas Sisargas & A Coruña & $43^{\circ} 21.13^{\prime}-43^{\circ} 22.40^{\prime} N-8^{\circ} 46.00^{\prime}-8^{\circ} 46.05^{\prime} \mathrm{O}$ & $80-96$ \\
\hline $173 \mathrm{~A}$ & Banco de Galicia & & $42^{\circ} 42.37^{\prime}-42^{\circ} 43.00^{\prime} \mathrm{N}-11^{\circ} 47.87^{\prime}-11^{\circ} 45.78^{\prime} \mathrm{O}$ & $769-760$ \\
\hline
\end{tabular}


Apéndice 2.-Bibliografía del catálogo. Se muestra la correspondencia entre la referencia numérica del listado de serpúlidos íbero-baleares presentado y las publicaciones pertinentes. Al haber utilizado los trabajos de Campoy (1982) y Ariño (1987) como base, gran parte de las referencias pertenecen a sus listados ibéricos. Los trabajos consultados para la realización de este catálogo se indican con un asterisco.

Appendix 2.-References of the catalogue. The correspondence between the numeral listing of the lberian-Balearic serpulids presented and relevant publications is shown. Having used the work of Campoy (1982) and Ariño (1987) as a basis, much of the lberian references belong to their listings. Studies consulted for the realization of this catalog are indicated with an asterisk.

1-Acuña, R., Durán, C., Rodríguez, M. \& Sanjuán, A., 1984. Campañas de estudio del macrobentos infralitoral rocoso en el parque natural de las islas Cíes (N. W. de España). I. Material, métodos y sistemática. Actas IV Simposio GIEB, Lisboa: 271-285.

2-Agirrezabalaga, F., 1980. Contribución al estudio de la fauna de Anélidos Poliquetos de las costas de Guipúzcoa. Tesis de Licenciatura. Universidad de Navarra.

3-Agirrezabalaga, F., 1984. Contribución al estudio de los Anélidos Poliquetos de la costa de Guipúzcoa. Munibe, 36: 119-130.

4-Aguirre, O., 1986. Estudio de la taxocenosis de Anélidos Poliquetos de las concreciones calcáreas del alga Mesophyllum lichenoides (Corallinacea, Rhodophyta) en Nerja, Málaga. Tesis de Licenciatura. Universidad Autónoma de Madrid.

5-Aguirrezabalaga, F., Altuna, A., Martínez de Murguia, A., Romero, A., Zaballa, K. \& Ibáñez, M., 1987. Contribución al conocimiento de la fauna marina de la Costa Vasca. V. Lurralde: Investigación y Espacio, 10: 109-128.

6-Alós, C. Especies capturadas en diversas estaciones del litoral Norte de la península del cabo de Creus. Resultados provisionales en elaboración.

7-*Alós, C., 1980. Contribución al estudio de los Anélidos Poliquetos endobiontes de esponjas. Departamento de Zoología, Facultad de Biología, Universidad de Navarra.

8-Alós, C., 1983. Anélidos Poliquetos del Cabo de Creus. II. Hojas de Posidonia oceanica. Publicaciones del Departamento de Zoología, Universidad de Barcelona, 9: 23-30.

9-*Alós, C., 1988. Anélidos Poliquetos del Cabo de Creus (Alt Empordá). Tesis Doctoral. Universidad de Barcelona. 838 pp.

10-Alós, C., Campoy, A. \& Pereira, F., 1982. Contribución al estudio de los Anélidos Poliquetos endobiontes de esponjas. Actas II Simpososio Ibérico de Estudios del Bentos Marino, 3: 139-157.

11-Alós, C. \& Pereira, F., 1989. Estudio de la población de Anélidos Poliquetos de Posidonia oceanica. Posidonia Newsletter, 2(1): 5-16.

12-Alquézar, E., 1980. Anélidos poliquetos de las formaciones de Dendropoma petraeum de las costas del sureste de España (Mediterráneo occidental). Tesis de Licenciatura. Universidad de Navarra.

13-Altuna, A., 1983. Contribución al conocimiento de la fauna marina de la costa de Guipúzcoa I. Lurralde: Investigación y Espacio, 6: 127-155.

14-Amoureux, L., 1972. Annélides Polychètes recuillies sur les pentes du talus continental au large de la Galice (Espagne). Campagnes 1967 et 1968 de la "Thalassa". Cahiers de Biologie Marine, 13: 63-89.

15-Amoureux, L., 1973. Annélides Polychètes recuilliees sur les pentes du talus continental au nord de la côte espagnole. Campagne 1970 de la "Thalassa". Cahiers de Biologie Marine, 14: 429-452.

16-Amoureux, L., 1974. Annélides Polychètes recuilles sur les pentes du talus continental au nord-ouest de l'Espagne \& du Portugal (Campagne 1972 de la "Thalassa"). Cuadernos de Ciencias Biológicas, Universidad de Granada, 3: 121-154.

17-Amoureux, L., 1976. Annélides Polychetes recoltes par J. Stirn en 1969, sur les cotes marocaines du Détroit de Gibraltar.
Cuadernos de Ciencias Biológicas, Universidad de Granada, 5: 5-33.

18-Amoureux, L. \& Calvario, J., 1981. Annélides Polychetes du Portugal. Données nouvelles. Arquivos do Museu Bocage. Série B, Notas, 1(12): 145-156.

19-Anadón, N., 1981. Contribución al conocimiento de la fauna bentónica de Vigo. III. Estudio de los arrecifes de Sabellaria alveolata (L.) (Polychaeta, Sedentaria). Investigación Pesquera, 45(1): 195-122.

20-Anadón, R., Gili, C., Guasch, E., Olivella, I., Polo, L., Ros, J., 1979. Distribución del poblamiento bentónico de una zona intermareal de la costa cantábrica gallega. Actas I Simposio Ibérico de Estudio del Bentos Marino: 673-710.

21-Angulo, R., Campoy, A. \& Ibáñez, M., 1978. Ecología de la costa guipuzcoana I. Bulletin du Centre d'Études et de Recherches Scientifiques Biarritz, 12(1): 157-184.

22-Arias, E. \& Morales, E., 1963. Ecología del Puerto de Barcelona y desarrollo de adherencias orgánicas sobre embarcaciones. Investigación Pesquera, 24: 139-163.

23-Arias, E. \& Morales, E., 1965. Ecología del Puerto de Barcelona y desarrollo de adherencias orgánicas sobre plantas sumergidas. Investigación Pesquera, 28: 44-79.

24-Arias, E. \& Morales, E., 1969. Ecología del Puerto de Barcelona y desarrollo de adherencias orgánicas sobre plantas sumergidas los años 1964-1965. Investigación Pesquera, 33: 179-200.

25-Arias, E. \& Morales, E., 1979. Variación estacional de organismos adherentes en el Puerto de Castellón de la Plana. Investigación Pesquera, 43(2): 353-384.

26-Baratech, L., 1984. Contribución al conocimiento de los Anélidos Poliquetos de las costas andaluzas. Tesis de Licenciatura. Universidad Autónoma de Madrid.

27-*Baratech, L. \& San Martín, G., 1987. Contribución al conocimiento de los Anélidos Poliquetos (Annelida: Polychaeta) de las costas andaluzas. Boletín del Instituto Español de Oceanografia, 4(2): 37-48.

28-*Baratech, L., San Martín, G. \& Aguirre, O., 1985. Presencia de Vermiliopsis monodiscus Zibrowius, 1967 (Polychaeta: Serpulidae) en las costas españolas. Miscelánea Zoológica, 9: 389-390.

29-Bellan, G., 1959. Campagnes de la "Calypso": Mer d'Alboran. 2, Annélides Polychètes. Annales de l'Institut Océanographique, 37: 315-342.

30-Bellan, G., 1960. Résultats scientifiques de la Campagne du N. R. P. FAIAL (1957). 2, Annélides Polychètes. Gabinete de Estudos das pescas. Lisboa. $31 \mathrm{pp}$.

31-*Bianchi, C. N., 1981. Policheti Serpuloidei. Guide per il riconoscimento delle specie animali delle acque lagunari e costiere italiane, 5: 1-87.

32-*Bianchi, C. N., Chessa, L. A. \& Morri, C., 1987. Serpuloidea (Annelida, Polychaeta) della Sardegna, con particolare riguardo alle lagune costiere. Atti del VII Convegno del Grupo G. Gadio per l'Ecologia di Base Cagliari 27-29 aprile 1984. Ecologia della Sardegna e delle isole Tirreniche. Supplemento al volume LIV (1984). 
33-Boscá, A., 1916. Fauna valenciana. Barcelona.

34-Camp, J., 1976. Comunidades bentónicas de sustrato duro del litoral N. E. español. IV. Poliquetos. Investigación Pesquera, 40(2): 533-550.

35-*Camp, J. \& Viñolas, C., 1984. Capítulo 25: Els Poliquets de les Illes Medes. En: Els Sistemes naturals de les illes Medes. Ros, J., Olivella, I. \& Gili, J. M. (eds.). Institut d'Estudis Catalans. Barcelona

36-Campoy, A., 1974. Contribución al estudio de la fauna de Anélidos Poliquetos de las costas españolas. Tesis de Licenciatura Universidad de Navarra.

37-Campoy, A., 1979. Lista de especies de Anélidos Poliquetos conocidas de las costas de la Península Ibérica. Investigación Pesquera, 43(3): 737-766.

38-*Campoy, A. 1981. Fauna de anélidos poliquetos de la Península Ibérica. Tesis doctoral. Universidad de Navarra. $759 \mathrm{pp}$.

39-*Campoy, A., 1982. Fauna de España. Fauna de Anélidos poliquetos de la Península Ibérica (II). Publicaciones de Biología de la Universidad de Navarra, Serie Zoología, 7: 1-781.

40-Campoy, A. \& Alquézar, E., 1982. Anélidos Poliquetos de las formaciones de Dendropoma petraeum (Monterosato) de las costas del sureste de España. Actas II Simposio Ibérico de Estudios del Bentos Marino, 3: 121-137.

41-Campoy, A. \& Jordana, R., 1978. Contribución a la fauna de Anélidos Poliquetos de las costas españolas: Nota sobre una relación de especies capturadas en Blanes (Gerona) y Aguilas (Murcia). Boletín de la Real Sociedad Española de Historia Natural (Sección Biológica), 76(1): 49-56.

42-Capaccioni, R., 1983. Anélidos Poliquetos del Mar Menor: Faunística y Ecología. Tesis de Licenciatura. Universidad de Valencia.

43-*Capaccioni, R., 1987. Anélidos Poliquetos de la ensenada de los Alfaques (Delta del Ebro, Mediterráneo Occidental). Tesis Doctoral. Universidad de Valencia. 534 pp.

44-*Capaccioni, R., García, M. \& Rodríguez, C., 1987. Anélidos Poliquetos del Mar Menor (S. E. España): inventario faunístico y caracterización ecológica y bionómica. Cahiers de Biologie Marine, 28: 403-428.

45-Carvalho, R. N. de, 1929. Catálogo de Colecçao de Invertebradosde Portugal existente no Museo Zoológico da Universidade de Coimbra. Vermes. I. Polychaeta. Memórias e Estudos do Museu Zoológico da Universidade de Coimbra, 37: 1-16.

46-Cuadras, J. \& Pereira, F., 1977. Invertebrates associated with Dardanus arrosor (Anomura, Diogenidae). Vie et Milieu, 27(3A): 301-310.

47-Desbruyeres, D., Guille, A. \& Ramos, J. M., 1972. Bionomie benthi que du plateau continental de la côte catalane espagnole. Vie et Milieu, 23(2B): 335-366.

48-Fauvel, P., 1914. Annélides Polychètes non-pélagiques provenant des Campagnes de l'Hirondelle et de la Princesse Alice (1885-1910). Résultats des Campagnes Scientifiques accomplies sur son yatch, par Albert 1 ${ }^{\text {er }}$, 46: 1-432.

49-Fischer-Piette, E., 1951. Stations de l'Annélide tubicole Mercierella enigmatica Fauvel sur la côte Nord d'Espagne. Bulletin du Laboratoire Maritime de Dinard, 34: 7-9.

50-Fischer-Piette, E., 1955. Répartition le long des côtes septentrionales de l'Espagne des principales espèces peuplant les roches intercotidaux. Annales de l'Institut Océanographique, 31: 37-124.

51-Fischer-Piette, E. \& Seoane, J., 1963. Examen écologique de la Ría de Camariñas. Bulletin de l'Institut Océanographique de Monaco, 31: 37-124.

52-*Fornós, J. J., Forteza, V. \& Martínez-Taberner, A., 1997. Modern polychaete reefs in Western Mediterranean lagoons: Ficopomatus enigmaticus (Fauvel) in the Albufera of Menorca, Balearic Islands. Paleogreography, Paleoclimatology, Paleoecology, 128: 175-186.

53-García Carrascosa, A. M., 1979. Contribución al conocimiento del bentos del mar Menor: Poblamientos bentónicos de las islas Perdiguera, Redonda y del Sujeto. Estudio descriptivo y cartografía bionómica. Actas I Simposio Ibérico de Estudios del Bentos Marino: 795-812.

54-García, O. L., García, M. A., Mora, J. \& Rodríguez, C., 1979. Contribución al estudio de los Anélidos Poliquetos del litoral gallego. Actas I Simposio Ibérico de Estudios del Bentos Marino: $161-171$

55-*García-Arberas, L. \& Rallo, A., 1994. Fauna béntica de los fondos de la Fosa del Capbreton (Golfo de Vizcaya. Atlántico Oriental): Anélidos Poliquetos. Cuadernos de Investigación Biológica (Bilbao), 18: 71-83.

56-*Garmendia, J. M., Sánchez Mata, A. \& Mora, J., 1998. Inventario de la macrofauna bentónica de sustratos blandos submareales de la Ría de Ares y Betanzos (NO de la Península Ibérica). Nova Acta Científica Compostelana (Bioloxía), 8: 209-231.

57-Gili, C., Anadón, R., Carbonell, J., Olivella, I. \& Ros, J., 1979. Comunidades bentónicas submarinas del litoral de Lugo. I. Resultados preliminares. Actas I Simposio Ibérico de Estudios del Bentos Marino: 711-750

58-Gili, J. M., Olivella, I., Zabala, M. \& Ros, J., 1979. Primera contribución al conocimiento del poblamiento bentónico de las cuevas submarinas del litoral catalán. Actas I Simposio Ibérico de Estudios del Bentos Marino: 813-836.

59-*Gómez, J. J. \& San Martín, G., 1985. Estudio faunístico-ecológico de las taxocenosis de Poliquetos y Moluscos de la Playa de Ribeira (Asturias). 1. Resultados faunísticos. Boletín del Instituto Español de Oceanografia, 2(3): 111-118.

60-*Gottfried Pillai, T. \& Ten Hove, H. A., 1994. On recent species of Spiraserpula Regenhardt, 1961, a serpulid polychaete hitherto known only from Cretaceous and Tertiary fossils. Bulletin of Natural History Museum Zoology Series, 60(1).

61-Graells, M., 1869. Exploración científica del Departamento maritimo de Ferrol. Fortanet. Madrid.

62-*Grémare, A., Sardá, R., Medernach, L., Jordana, E., Pinedo, S., Amoroux, J. M., Martin, D., Nozais, C. \& Charles, F., 1998. On the Dramatic Increase of Ditrupa arietina O. F. Müller (Annelida: Polychaeta) Along Both the French and the Spanish Coasts. Estuarine, Coastal and Shelf Science, 47: 447-457.

63-Gutiérrez, M. E., Rallo, A., Rodríguez, F. \& Saiz, 1979. Resultados preliminares del estudio de las comunidades bentónicas de sustrato duro establecidas en el litoral de Vizcaya. Actas I Simposio Ibérico de Estudios del Bentos Marino: 643-655.

64-*Harris, T. \& Knight-Jones, E. W., 1964. Spirorbis infundibulum n. sp. (Polychaeta: Serpulidae) from Tenaerea shelves on the Costa Brava. Annals and Magazine of Natural History, 13(7): 347-351.

65-Hartmann-Schröder, G., 1979. Die Polychaeten der "Atlantischen Kuppenfahrt" von F. S. "Meteor" (Fahrt 9 c, 1967) 1. Proben aus Schleppgeräten. Meteor-Forschungsergebnisse. Reihe D, Biologie, 31: 63-90.

66-Henke, J., 1972. Erganzende Bemerkungen zur Makrofauna der Spanischen Südküste. Zoologischer Anzeiger, 189(5/6): 348-351.

67-Herdocio Peralta, N., 1984. Fijación de organismos bentónicos marinos sobre superficies experimentales vírgenes: resultados preliminares. Actas IV Simposio GIEB, Lisboa, 1: 307-318.

68-Ibáñez Ártica, M., 1978. Carácterísticas biogeográficas del litoral de la costa vasca. Lurralde: Investigación y Espacio, 1: 121-127. 
69-Ibáñez, M., 1973. Catálogo de los Anélidos Poliquetos citados en las costas españolas. Cuadernos de Ciencias Biológicas, Universidad de Granada, 2(2): 121-140.

70-Ibáñez, M., Romero, A., Feliu, J., Borja, A., Altuna, A. \& Aguirrezabalaga, F., 1984. Consideraciones sobre la biogeografía de la costa vasca. Actas IV Simposio GIEB, Lisboa, 1: $47-56$

71-Ibáñez, M., Romero, A., San Vicente, C. \& Zaballa, K., 1984. La contaminación marina en Guipúzcoa II: Estudio de los indicadores biológicos de la contaminación en los sedimentos de las Rías Urola, Oria y Urumea. Lurralde: Investigación y Espacio, 1984: 197-216.

72-Iraola, J. J. \& Ibáñez, M., 1976. Fauna marina de la costa vasca. Caja de Ahorros Municipal de San Sebastián. San Sebastián. 163 pp.

73-Knight-Jones, P. \& Knight-Jones, E. W., 1977. Taxonomy and ecology of British Spirorbidae (Polychaeta). Journal of the Marine Biological Association of the United Kingdom, 57: 453-499.

74-*Laborda, A. J., Cimas, E. \& Marcilla, I., 1991. Influencia de la contaminación en la distribución de los anélidos poliquetos del sustrato rocoso intermareal de la región del Cabo de Peñas (Asturias, España). Boletín del Instituto Español de Oceanografía, 7(1): 75-90.

75-*Lombas, I. \& Anadón, N., 1985. Estudio de la fauna de microhábitats esciáfilos intermareales de Luanco (Asturias). Revista de Biología, Universidad de Oviedo, 3: 107-120.

76-*López, E., 1995. Anélidos Poliquetos de sustratos duros de las Islas Chafarinas. Tesis Doctoral. Universidad Autónoma de Madrid. 672 pp.

77-*López, L., 1999. Estudio de la macrofauna bentónica de la desembocadura del rio Piedras (Huelva). Tesis Doctoral. Universidad Complutense de Madrid. 438 pp.

78-López-Jamar, E., 1978. Macrobentos infaunal de la Ría de Pontevedra. Boletín del Instituto Español de Oceanografia, 4(4): 111-130.

79-López-Jamar, E., 1979. Estructura de la infauna bentónica de la Ría de Pontevedra. Actas I Simposio Ibérico de Estudios del Bentos Marino: 751-780.

80-López-Jamar， E., Iglesias，J., Otero, J. J., 1984. Contribution of infauna and mussel-raft epifauna to demersal fish diets. Marine Ecology Progress Series, 15: 13-18.

81-Maluquer, J., 1915. Excursió oceanográfica en la costa de l'Empordá. Butlletí de la Institució Catalana d'Història Natural, 15: 87.

82-Maluquer, J., 1916. Treballs oceanográfics en la costa de l'Empordá. Annuari Junta de Ciencies Naturals Barcelona, 1: 221-240.

83-Margalef, R., 1948. Miscelánea de Zoología dulceacuícola. II. Publicaciones del Instituto de Biología Aplicada, 5: 69-76.

84-*Marques, J. C., Rodrigues, L. B. \& Nogueira, A. J. A., 1993. Intertidal macrobenthic communities structure in the Mondego Estuary (Western Portugal): Reference situation. Vie et Milieu, 43(2-3): 177-187.

85-Martín, D., 1986. Algunos datos interesantes sobre la fauna de anélidos poliquetos asociados a las concreciones de algas calcáreas. Actas V Simposio Ibérico de Estudios del Bentos Marino, Tenerife.

86-Martín, D., 1987. La comunidad de Anélidos Poliquetos de las concreciones de algas calcáreas del litoral catalán. Caracterización de las especies. Publicaciones del Departamento de Zoología, Universidad de Barcelona, 13: 45-54.

87-Martín, D. 1986. Anélidos Poliquetos y moluscos asociados a algas calcáreas. Tesis de Licenciatura. Universidad de Barcelona.
88-Martín, D. \& Sardá, R. Lista de Poliquetos de Gibraltar y Baleares. Resultados provisionales en elaboración.

89-*Méndez, N. \& Cardell, M. J., 1996. Littoral annelid polychaetes inhabiting soft bottoms of the Barcelonès (Catalonia, NE Spain). Miscel-lània Zoològica, 19(1): 119-147.

90-Morales, E. \& Arias, E., 1964. Ecología del puerto de Barcelona y desarrollo de adherencias orgánicas sobre placas sumergidas. Investigación Pesquera, 28: 49-79.

91-Morales, E. \& Arias, E., 1977. Variación estacional de organismos adherentes en la bahía de Escombreras (SE de España). Investigación Pesquera, 41(2): 473-500.

92-Morales, E. \& Arias, E., 1979. Estudio comparativo del "fouling" en los puertos de Barcelona, Castellón de la Plana y Escombreras. Informes Técnicos del Instituto de Investigaciones Pesqueras, 62: 1-18.

93-Morales, E. \& Arias, E., 1979. Variación estacional del fouling en el Puerto de Guetaria (Norte de España). Investigación Pesquera, 43(2): 385-400.

94-*Moreira, J., 1999. Anélidos Poliquetos de sustratos blandos infralitorales de la ensenada de Baiona (Galicia). Memoria de Licenciatura. Universidad de Vigo.

95-*Moreira, J., Quintas, P. \& Troncoso, S. J., 2006. Spatial distribution of soft-bottom polychaete annelids in the Ensenada de Baiona (Ría de Vigo, Galicia, north-west Spain). Scientia Marina, 70(S3): 217-224.

96-Moreno Twose, A., 1985. Estructura de las comunidades asociadas al mejillón (Mytilus edulis L.) en Sopelana (Costa Vasca). Tesis de Licenciatura. Universidad de Navarra.

97-Nobre, A., 1903. Subsidios para o estudio da fauna marinha do sul de Portugal. Anais de Sciências Naturais, 8: 153-160.

98-Nobre, A., 1937. Fauna marinha de Portugal. $1^{\text {er }}$ aditamiento. Memórias e Estudos do Museu Zoológico da Universidade de Coimbra, Série I, 99: 1-30.

99-Nobre, A., 1942. Notas sobre a fauna des ilhas Berlingas e Farilhoes. Memórias e Estudos do Museu Zoológico da Universidade de Coimbra, Série I, 138: 45-46.

100-Ocharan, F. J., 1978. Primera cita para la Península Ibérica de Phoronis hippocrepia Wright 1856 (Phoronida). Revista de la Facultad de Ciencias, Universidad de Oviedo, 17-19: 393-397.

101-*Parapar, J., 1991. Anélidos Poliquetos bentónicos de la Ría de Ferrol (Galicia). Tesis Doctoral. Universidad de Santiago de Compostela. 1104 pp.

102-*Parapar, J., Besteiro, C. \& Urgorri, V., 1992. Nuevas aportaciones al conocimiento de los anélidos poliquetos en el litoral gallego (N. O. Península Ibérica). Nova Acta Científica Compostelana (Bioloxía), 3: 109-123.

103-*Parapar, J., Besteiro, C. \& Urgorri, V., 1993. Aportaciones a la taxonomía y autoecología de los anélidos de la península ibérica: poliquetos de la Ría de Ferrol. Cahiers de Biologie Marine, 34: 411-432.

104-*Parapar, J., Besteiro, C. \& Urgorri, V., 1996. Inventario dos Poliquetos (Annelida: Polychaeta) de Galicia. Cadernos da Área de Ciencias Biolóxicas (Inventarios). Seminario de Estudos Galegos, XVI. Ed. do Castro, 178 pp.

105-*Parapar, J., Besteiro, C., Urgorri, V. \& Troncoso, J. S., 1994. Aportaciones al género Spirorbis Daudin, 1800 (Annelida, Polychaeta) en la Península Ibérica. Boletín de la Real Sociedad Española de Historia Natural (Sección Biológica), 91(1-4): 41-48.

106-*Parapar, J., Fernández, L., González-Gurriarán, E. \& Muíño, R., 1997. Epibiosis and masking material in the spider crab Maja squinado (Decapoda: Majidae) in the Ría de Arousa (Galicia, NW Spain). Cahiers de Biologie Marine, 38: 221-234.

107-*Parapar, J., San Martín, G., Urgorri, V. \& Besteiro, C., 1993. Anélidos Poliquetos mesopsámmicos sublitorales de la costa de Ceuta (España). Cahiers de Biologie Marine, 34: 363-381. 
108-*Planas, M., 1986. Poliquetos de los fondos rocosos de las Islas Cíes (NW España). Trabajos Compostelanos de Biología, 13: 71-88.

109-*Porras, R., Bataller, J. V., Murgui, E. \& Torregrosa, M. T., 1996. Trophic structure and community composition of polychaetes inhabiting some Sabellaria alveolata (L.) reefs along the Valencia Gulf coast, western Mediterrranean. Marine Ecology, 17(4): 583-602.

110-Pruvot, G., 1895. Coup d'oeil sur la distribution générale des Invertébrés dans la région de Banyuls (golfe du Lion). Archives de Zoologie Expérimental et Générale, 3(3): 629-658.

111-Pruvot, G., 1901. Le Roland et sa première croisière para la côte de Catalogne en juillet-aôut 1900. Archives de Zoologie Expérimental et Générale, 9: 40.

112-Quatrefages, A., 1865. Histoire Naturelle des Annelides d'eau douce et marins. Roret. Paris. T. 1, 588 p.; T. 2, 336 p.; T. 3, 337-794 p.

113-*Quintino, V. \& Gentil, F., 1987. Etude faunistique et coenotique de la faune annélidienne des lagunes d'Albufeira et Obbidos (Portugal). Cahiers de Biologie Marine, 28: 59-72.

114-*Rallo, A., 1988. Anélidos Poliquetos recogidos en la plataforma litoral vizcaína (Golfo de Vizcaya, Atlántico Oriental). I. Resultados taxnómicos. Cuadernos de Investigación Biológica (Bilbao), 13: 135-143.

115-*Rallo, A., García-Arberas, L. \& Isasi, I., 1993. Fauna macrobéntica de los fondos del cañón de Capbretón: análisis faunístico de poliquetos, crustáceos y cnidarios y caracterización de puntos de muestreo según estos descriptores. Cahiers de Biologie Marine, 35: 69-90.

116-*Redondo, M. S. \& San Martín, G., 1997. Anélidos Poliquetos de la costa comprendida entre el cabo de San Antonio y el puerto de Valencia (Mediterráneo occidental). Publicaciones Especiales, Instituto Español de Oceanografia, 23: 225-233.

117-Rioja, E., 1916. Nota de algunos anélidos recogidos en las costas de Gijón y San Vicente de la Barquera. Boletín de la Real Sociedad Española de Historia Natural, 16: 462-466.

118-Rioja, E., 1917. Datos para el conocimiento de la Fauna de Anélidos Poliquetos del Cantábrico. Trabajos del Museo Nacional de Ciencias Naturales, Serie Zoológica, 29: 1-111.

119-Rioja, E., 1917. Nota sobre algunos Anélidos Poliquetos recogidos en Málaga. Boletín de la Real Sociedad Española de Historia Natural, 17: 176-185.

120-Rioja, E., 1917. Nota sobre algunos anélidos interesantes de Santander. Boletín de la Real Sociedad Española de Historia Natural, 17: 221-228.

121-Rioja, E., 1917. Nota sobre una excursión por las costas de Gijón. Boletín de la Real Sociedad Española de Historia Natural, 17: 488-494.

122-*Rioja, E., 1918. Adiciones a la fauna de Anélidos Poliquetos del Cantábrico. Revista de la Real Academia de Ciencias Exactas, Físicas y Naturales de Madrid, 17: 54-80.

123-Rioja, E., 1918. Datos para el conocimiento de la Fauna de Anélidos Poliquetos del Cantábrico (II parte). Trabajos del Museo Nacional de Ciencias Naturales, Serie Zoológica, 37: $1-99$

124-*Rioja, E., 1919. Una curiosa anomalía de Hydroides norvegica Gunnerus y algunas consideraciones acerca de la filogenia de los serpúlidos. Boletín de la Real Sociedad Española de Historia Natural, 19: 445-449.

125-Rioja, E., 1920. Una campaña biológica en el Golfo de Valencia. Anales del Instituto General y Técnico de Valencia, 20: $1-36$.

126-Rioja, E., 1923. Estudio sistemático de las especies ibéricas del Suborden Sabelliforma. Trabajos del Museo Nacional de Ciencias Naturales, Serie Zoológica, 48: 1-144.
127-Rioja, E., 1923. Algunas especies de Anélidos Poliquetos de las costas de Galicia. Boletín de la Real Sociedad Española de Historia Natural, 23: 333-345.

128-Rioja, E., 1924. La Mercierella enigmatica Fauvel, serpúlido de agua salobre, en España. Boletín de la Real Sociedad Española de Historia Natural, 24: 160-169.

129-Rioja, E., 1925. Anélidos Poliquetos de San Vicente de la Barquera. Trabajos del Museo Nacional de Ciencias Naturales, Serie Zoológica, 53: 1-62.

130-*Rioja, E., 1931. Estudio de los Poliquetos de la Península Ibérica. Memorias de la Real Academia de Ciencias Exactas, Físicas y Naturales de Madrid, Serie de ciencias naturales, 2: $1-471$.

131-*Rioja, E., 1935. Anélidos Poliquetos de las campañas del Instituto Español de Oceanografía. Trabajos del Instituto Español de Oceanografía, 13: 1-44.

132-*Rodríguez, A. M. \& Viéitez, J. M., 1992. Macrofauna bentónica de los primeros metros del piso infralitoral de la costa de Punta Umbría (Huelva). Boletín del Instituto Español de Oceanografia, 8(2): 327-338.

133-Rodríguez, F., Gutiérrez, M. E., Rallo, A. \& Saiz, I., 1979. Actas I Simposio Ibérico de Estudios del Bentos Marino: 149-161.

134-Roule, L., 1896. Résultats scientifiques de la Campagne du "Caudan" dans le golf de Gascogne. Annélides. Annales de l' $U$ niversité de Lyon, 26: 439-472.

135-Rubio, M., 1971. Contribución al estudio de la fauna bentónica del litoral de Blanes (Gerona). Res. Tesis doctoral. Universidad de Barcelona. 20 pp.

136-*Sáiz, J. I. \& Urkiaga, J., 1997. Comunidades faunísticas en el intermareal del abra de Bilbao. Publicaciones Especiales, Instituto Español de Oceanografia, 23: 121-131.

137-Saldanha, L., 1974. Estudo do povoamento dos horizontes superiores da rocha litoral da costa Arrábida (Portugal). Arquivos do Museu Bocage, $2^{a}$ serie, 5(1): 1-382.

138-Saldanha, L., 1984. Fauna Submarina Atlântica. Publicações Europa-America. 180 pp.

139-*San Martín, G. \& Viéitez, J. M., 1981. Contribución al conocimiento de los Anélidos Poliquetos epibiontes de la Bahía de Palma de Mallorca. Actas I Simposio Ibérico de Estudios del Bentos Marino, 1: 193-204.

140-*San Martín, G., Acero, M. I., Contonente, M., Gómez, J. J., 1982. Una colección de anélidos poliquetos de las costas mediterráneas andaluzas. Actas II Simposio Ibérico de Estudios del Bentos Marino, 3: 171-182.

141-*San Martín, G. \& Aguirre, O., 1991. Ciclo anual de los Poliquetos asociados al alga Mesophyllum lichenoides (Ellis) en una playa mediterránea. Boletín del Instituto Español de Oceanografia, 7(1): 157-170.

142-*San Martín, G. \& Alvarado, R., 1982. Nota sobre Poliquetos de la Isla de Cabrera (Baleares). Boletín de la Real Sociedad Española de Historia Natural (Sección Biológica), 79: 221-234.

143-*San Martín, G., Estapé, S., García-Ocejo, A., Gómez, C. \& Jiménez, P., 1990. Estudio de la taxocenosis de anélidos poliquetos de rizomas de Posidonia oceanica en las costas de Almería. Boletín del Instituto Español de Oceanografía, 6(1): 41-58.

144-*San Martín, G., López, E., 2004. Capítulo 11: Poliquetos. En: Rodríguez, F. (ed.) Proyecto Andalucía: Fauna Andaluza. Publicaciones Comunitarias. Sevilla.

145-*San Martín, G. \& Viéitez, J. M., 1981. Contribución al conocimiento de los anélidos poliquetos epibiontes en la bahía de Palma de Mallorca. Actas I Simposio Ibérico de Estudios del Bentos Marino: 193-205. 
146-*San Martín, G. \& Viéitez, J. M., 1984. Anélidos Poliquetos de los rizomas de Posidonia oceanica en las costas del Cabo de Palos (Murcia, España). I International Workshop Posidonia oceanica beds, 1: 149-157.

147-*San Martín, G. \& Viéitez, J. M., 1991. Catálogo de los anélidos poliquetos del Museo Nacional de Ciencias Naturales de Madrid. Boletín de la Real Sociedad Española de Historia Natural (Sección Biológica), 87(1-4): 93-131.

148-Sanz, A., 1982. Sistemática y ecología del intermareal de Ondaterra (San Sebastián). Anélidos Poliquetos. Tesis de Licenciatura. Universidad de Navarra.

149-*Sardá, R., 1982. Anélidos Poliquetos de la bahía de Algeciras. Nota sobre una colección próxima a la desembocadura del río Guadarranque. Actas II Simposio Ibérico de Estudios del Bentos Marino, 3: 165-170.

150-Sardá, R., 1984. Estudio sobre la fauna de anélidos poliquetos de las zonas mediolitoral e infralitoral, en la región del Estrecho de Gibraltar. Tesis Doctoral. Universidad de Barcelona. $901 \mathrm{pp}$.

151-Sardá, R., 1985. Estudio sobre la fauna de anélidos poliquetos de las zonas mediolitoral e infralitoral, en la región del Estrecho de Gibraltar. Res. Tesis Doctoral. Universidad de Barcelona. 49 pp.

152-Sardá, R., 1986. Contribución al conocimiento de las poblaciones anelidanas infaunales de la costa catalana. Publicaciones del Departamento de Zoología, Universidad de Barcelona, 12: 27-36.

153-Seoane, V. L., 1886. Reseña de la Historia Natural de Galicia. Lugo.

154-*Serrano, L. G., Cardell, M. J., Lozoya, J. P. \& Sardá, R., 2011. A polychaete-dominated community in the NW Mediterranean Sea, 20 years after cessation of sewage discharges. Italian Journal of Zoology, 78 (S1): 333-346.

155-Sola, J. C., 1985. Estudio cualitativo y cuantitativo de los Anélidos Poliquetos del Estuario del Bidasoa. Tesis de Licenciatura. Universidad de Navarra.

156-Templado, J., García-Carrascosa, M., Baratech, L., Capaccioni, R., Juan, A., Lopez-Ibor, A. \& Silvestre, R., 1986. Estudio preliminar de la fauna asociada a los fondos coralíferos del mar de Alborán. Boletín del Instituto Español de Oceanografía, 3(4): 93-104.

157-*Tena, J., 1996. Faunística y Ecología de los Anélidos Poliquetos de los fondos de Sustrato Duro del Archipiélago de las Chafarinas (S. W. Mar de Alborán). Tesis Doctoral. Universidad de Valencia. $549 \mathrm{pp}$.

158-*Tena, J., Capaccioni-Azzati, R., Torres-Gavila, F. J. \& García-Carrascosa, A. M., 2000. Polychaetes associated with diferent facies of the photophilic algal community in the Chafarinas Archipielago (SW Mediterranean). Bulletin of Marine Science, 67(1): 55-72.

159-*Torres, F. J., 2007. Estudio faunístico, ecológico y ambiental de la fauna de Anélidos Poliquetos de sustratos sueltos de las islas Chafarinas (Mar de Alborán, S. W. Mediterráneo). Tesis Doctoral. Universidad de Valencia. $720 \mathrm{pp}$.

160-Viéitez, J. M., 1978. Comparación ecológica de dos playas de las Rías de Ponteverdra y Vigo. Tesis Doctoral. Universidad Complutense de Madrid. 273 pp.

161-Viéitez, J. M., 1979. Ecología comparada de dos playas de las Rías de Pontevedra y Vigo. Fundación Juan March, Serie Universitaria, 91. Madrid. $46 \mathrm{pp}$.
162-Viéitez, J. M., 1981. Estudio de las comunidades bentónicas de dos playas de las Rías de Pontevedra y Vigo (Galicia, España). Boletín del Instituto Español de Oceanografia, 6: 242-258.

163-Villalba, A. \& Viéitez, J. M., 1985. Estudio de la fauna de anélidos poliquetos de sustrato rocoso intermareal de una zona contaminada de la Ría de Pontevedra (Galicia). Resultados biocenóticos. Cahiers de Biologie Marine, 26: 359-377.

164-Zaballa, K., 1985. Taxocenosis Anelidiana en Estuarios de la Provincia de Guipúzcoa. Tesis de Licenciatura. Universidad del País Vasco.

165-*Zenetos, A., Gofas, S., Verlaque, M., Cinar, M. E., García Raso, J. E., Bianchi, C. N., Morri, C., Azzurro, E., Bilecenoglu, M., Froglia, C., Siokou, I., Violanti, D., Sfriso, A., San Martín, G., Giangrande, A., Katagan, T., Ballesteros, E., Ramos-Esplá, A., Mastrototaro, F., Ocaña, O., Zingone, A., Gambi, M. C. \& Streftaris, N., 2010. Alien species in the Mediterranean Sea by 2010. A contribution to the application of European Union's Marine Strategy Framework Directive (MSFD). Part I. Spatial distribution. Mediterranean Marine Science, 11(2): 381-493.

166-Zibrowius, H., 1968. Etude morphologique, systématique et écologique des Serpulidae (Annelida: Polychaeta) de la région de Marseille. Recueil des Travaux de la Station Marine d'Endoume, 45(59): 81-252.

167-Zibrowius, H., 1969. Serpulidae (Annelida: Polychaeta) des campagnes du "Skagerrak" (1946) et du "Faial" (1957) au large du Portugal. Boletim da Sociedade Portuguesa de Ciências Naturais, 12: 117-131.

168-*Zibrowius, H., 1969. Review of some little known genera of Serpulidae (Annelida: Polychaeta). Smithsonian Contributions to Zoology, 42: 1-22.

169-*Zibrowius, H., 1970. Les especes méditerranéennes du genre Hydroides (Polychaeta: Serpulidae). Remarques sur le prétendu polymorphisme de Hydroides unicnata. Téthys, 2(3): 691-746.

170-Zibrowius, H., 1971. Revision of Metavermilia Bush (Polychaeta: Serpulidae) with description of three new from off Portugal, Gulf of Guinea and western Indian Ocean. Journal of the Fisheries Research Board of Canada, 28: 1373-1383.

171-*Zibrowius, H., 1973. Remarques sur trois espèces de Serpulidae aclimatées en Méditerranée: Hydroides dianthus (Verrill 1873), H. dirampha Mörch 1863 et H. elegans Haswell 1883. Rapports et Procès Verbaux des Réunions, Commission Internationale pour l'Exploration Scientifique de la Mer Méditerranée, 21(9): 683-686.

172-*Zibrowius, H., 1983. Spirorbis marioni et Pileolaria berkeleyana, spirorbidae exotiques dans les ports de la Méditerranée occidentale (suite). Rapports et Procès Verbaux des Réunions, Commission Internationale pour l'Exploration Scientifique de la Mer Méditerranée, 28(3): 255-256.

173-*Zibrowius, H. \& Bianchi, C. N., 1981. Spirorbis marioni et Pileolaria berkeleyana, spirorbidae exotiques dans les ports de la Méditerranée nord-occidentale. Rapports et Procès Verbaux des Réunions, Commission Internationale pour l'Exploration Scientifique de la Mer Méditerranée, 27(2): 162-163.

174-*Zibrowius, H., Ten Hove, H. A., 1987. Neovermilia falcigera (Roule, 1898) A Deep- and cold-water Serpulid Polychaete Common in the Mediterranean Plio-Pleistocene. Bulletin of the Biologial Society of Washington, 7: 259-271. 